DESY 10-079

June 2010

\title{
Coupled scalar fields in a flat FRW universe: renormalisation
}

\author{
Jürgen Baacke ${ }^{1}$, Laura Covi², Nina Kevlishvili²,3 \\ ${ }^{1}$ Fakultät Physik, Technische Universität Dortmund \\ D - 44221 Dortmund, Germany \\ ${ }^{2}$ Deutsches Elektronen Synchrotron DESY \\ D - 22603 Hamburg, Germany, \\ ${ }^{3}$ Andronikashvili Institute of Physics, GAS, 0177 Tbilisi, Georgia
}

\begin{abstract}
We study the non-equilibrium dynamics of a system of coupled scalar fields in a Friedmann-Robertson-Walker (FRW) universe. We consider the evolution of spatially homogeneous "classical" fields and of their quantum fluctuations including the quantum backreaction in the one-loop approximation. We discuss in particular the dimensional regularisation of the coupled system and a special subtraction procedure in order to obtain the renormalised equations of motion and the renormalised energy-momentum tensor and ensure that the energy is well-defined and covariantly conserved. These results represent at the same time a theoretical analysis and a viable scheme for stable numerical simulations. As an example for an application of the general formalism, we present simulations for a hybrid inflationary model.
\end{abstract}

\section{Introduction}

While the renormalisation of a single scalar field in a Friedmann-RobertsonWalker (FRW) universe, and fermion fields coupled to such field, have been discussed by various authors [1, 2, 3, 4, 5, 6, a consistent and coherent formulation of coupled scalar field models including non-minimal gravity couplings and full renormalisation is still missing. As a first step in that direction,

E-mails: juergen.baacke@tu-dortmund.de

laura.covi@desy.de nina.kevlishvili@desy.de 
we study here such a system of coupled fields in the one-loop approximation with special emphasis on its renormalisation in the $\overline{M S}$ scheme. This will allow us to obtain a consistent set of coupled equations of motion without divergences and numerical instabilities, which is highly suitable for numerical applications. Coupled scalar fields appear in cosmology in multi-field models of inflation and are important as well in particle physics in the context of grand unified theories, which contain several Higgs fields.

The standard example of a coupled system of scalar fields in cosmology is of course the hybrid model of inflation which has been introduced by Linde [7, 8, 9] and whose coupled dynamics at the end of the inflationary phase has received wide attention [10, 11, 12, 13, 14, 15, 16, 17, 18, 19, 20, 21, 22, 23, 24, 25] (for a recent review see [26]). Unlike other models for inflation and for preheating, where one has just one "inflaton" field with vacuum quantum numbers coupled to various other boson and fermion fields, this type of models has two scalar fields with can acquire a time dependent vacuum expectation value. So these fields and their quantum fluctuations can mix dynamically.

The renormalisation of such a coupled system of bosons out of equilibrium has been considered previously by Cormier et al. 222 in the one-loop approximation, and in Ref. [27] in the Hartree approximation. The one-loop approximation includes the quantum back-reaction of the quantum fluctuations on the classical fields, the Hartree approximation includes in addition the backreaction on the quantum fluctuation themselves. This approximation has been considered as well by Bastero-Gil et al. [13] with a momentum cutoff for the quantum fluctuations. These publications do not include the coupling of the fields to gravity, which may be a reasonable approximation in the period of preheating after inflation. However, when describing the evolution during inflation and the end of inflation, the inclusion of the coupling to gravity could be important and it is necessary for consistency, since those couplings are generated by quantum corrections. Moreover in recent years, such non-minimal gravitational coupling has drawn a lot of attention in the context of realising inflation within the Standard Model [28, 29] and it is surely an interesting issue to include it also in the case of many fields. In those models it became clear pretty soon that quantum effects on the inflation potential cannot be neglected [30, 31, 32] and it may be the same also for the hybrid case.

The one-loop renormalisation for the out-of-equilibrium evolution of a single scalar field in a FRW universe has been considered by several authors [1, 2, 15, 33, 34, 35, 36]. The technical approach which we will use here is based on Ref. [36, which itself uses a formalism developed in Ref. [37]. As in these references we establish here a "renormalised computation scheme", 
i.e., the renormalisation is taken into account with the same rigour as in more formal approaches, and at the same time the formalism can be implemented efficiently into a numerical code. In particular the renormalisation is independent of the initial conditions, though the divergent momentum integrals depend on the initial effective masses of the system. The energy-momentum tensor is in this formulation always finite and covariantly conserved.

The paper is organised as follows: in Sec. 2 we recall some basic relations of FRW cosmology, with special attention to an extension beyond space-time dimension $n=4$ and with the inclusion of higher dimensional gravity counterterms, which are needed in order to properly renormalise the coupled fields system; in Sec. 3 we define the general model whose dynamical equations we will formulate in the one-loop approximation; in Sec. 4 we discuss in detail the regularisation and renormalisation of the equation of motions and of the energy-momentum tensor. Finally in Sec. 5 we give few selected results to show the applicability of this scheme and the stability of the numerical results and then conclude. More exhaustive numerical results will be given in a forthcoming publication. Various technical details can be found in the Appendices A,

\section{Short review of FRW cosmology}

We consider here a spatially isotropic and flat space-time with $n-1$ spacial dimensions, which can be described by a FRW metric with $k=0$ curvature parameter as

$$
d s^{2}=d t^{2}-a^{2}(t) d \boldsymbol{x}^{2}
$$

where $d \boldsymbol{x}^{2}=\sum_{i=0}^{n-1} d x_{i}^{2}$. The effective action of the coupled gravity-scalar fields system is given by

$$
\begin{aligned}
\mathcal{S}= & \int d^{n} x \sqrt{-g}\left[\frac{M_{P}^{2}}{2} R+\Lambda g_{\mu \nu}+\delta \alpha R^{2}+\delta \beta R^{\alpha \beta} R_{\alpha \beta}+\delta \gamma R^{\alpha \beta \gamma \delta} R_{\alpha \beta \gamma \delta}\right] \\
& +\int d^{n} x \mathcal{L}\left(\Phi_{i}, g_{\mu \nu}, R\right) .
\end{aligned}
$$

The first two terms are the Einstein-Hilbert action and a cosmological constant term, which allows to tune the energy density of the ground state to zero. We have introduced also all the gravitational terms up to dimension four for later convenience. The last term is instead given by the action for the matter fields, in our case a system of coupled bosons described in the next section. 
The time evolution of the $a(t)$ is governed by the Einstein's field equation, which for the Einstein-Hilbert case $\delta \alpha=\delta \beta=\delta \gamma=0$ is given by

$$
G_{\mu \nu}+\Lambda g_{\mu \nu}=-8 \pi G<T_{\mu \nu}>
$$

where the general expression for energy-momentum tensor is

$$
T_{\mu \nu}(x)=\frac{2}{\sqrt{-g(x)}} \frac{\delta \mathcal{S}_{\text {matter }}}{\delta g_{\mu \nu}} .
$$

In quantum field theory renormalisation requires to introduce a counterterm action. Besides the usual mass and coupling constant counterterms the coupling to gravity induces divergences which require the inclusions of the higher curvature terms written in Eq. (2.2) and therefore a modification of the original Einstein field equations. These then take the form

$$
(1+\delta Z) G_{\mu \nu}+\delta \alpha^{(1)} H_{\mu \nu}+\delta \beta^{(2)} H_{\mu \nu}+\delta \gamma H_{\mu \nu}+(\Lambda+\delta \Lambda) g_{\mu \nu}=-8 \pi G<T_{\mu \nu}>\text {. }
$$

The tensors ${ }^{(1)} H_{\mu \nu},{ }^{(2)} H_{\mu \nu}$ and $H_{\mu \nu}$ are related to the variation of the higher curvature terms $R^{2}, R^{\alpha \beta} R_{\alpha \beta}$ and $R^{\alpha \beta \gamma \delta} R_{\alpha \beta \gamma \delta}$. Since gravity is not quantised, all divergences arise, at least technically, from the quantum fluctuations of the matter fields in the energy-momentum tensor. Therefore one may prefer to include the counterterms into a redefinition of the right hand side of the Einstein equations via

$$
T_{\mu \nu} \rightarrow T_{\mu \nu}+\delta \tilde{Z} G_{\mu \nu}+\delta \tilde{\alpha}^{(1)} H_{\mu \nu}+\delta \tilde{\beta}^{(2)} H_{\mu \nu}+\delta \tilde{\gamma} H_{\mu \nu}+(\tilde{\Lambda}+\delta \tilde{\Lambda}) g_{\mu \nu}
$$

Here $\delta \tilde{Z}=\delta Z / 8 \pi G$ and similarly for the other terms.

For the various tensors we use the conventions of Ref. [38]: the Einstein curvature tensor is defined as

$$
G_{\mu \nu}=R_{\mu \nu}-\frac{1}{2} g_{\mu \nu} R
$$

the Ricci tensor and the Ricci scalar are

$$
R_{\mu \nu}=R_{\mu \nu \lambda}^{\lambda}, \quad R=g^{\mu \nu} R_{\mu \nu},
$$

where the curvature tensor is given by

$$
R_{\alpha \beta \gamma}^{\lambda}=\partial_{\gamma} \Gamma_{\alpha \beta}^{\lambda}-\partial_{\alpha} \Gamma_{\gamma \beta}^{\lambda}+\Gamma_{\gamma \delta}^{\alpha} \Gamma_{\alpha \beta}^{\delta}-\Gamma_{\alpha \delta}^{\lambda} \Gamma_{\gamma \beta}^{\delta} .
$$


The higher curvature tensors are defined as 38

$$
\begin{aligned}
{ }^{(1)} H_{\mu \nu} & =\frac{1}{\sqrt{-g}} \frac{\delta}{\delta g^{\mu \nu}} \int d^{n} x \sqrt{-g} R^{2} \\
& =2 R_{; \mu \nu}-2 g_{\mu \nu} \square R-\frac{1}{2} g_{\mu \nu} R^{2}+2 R R_{\mu \nu} \\
& =\frac{1}{\sqrt{-g}} \frac{\delta}{\delta g^{\mu \nu}} \int d^{n} x \sqrt{-g} R^{\alpha \beta} R_{\alpha \beta} \\
& =2 R_{\mu ; \nu \alpha}^{\alpha}-\square R_{\mu \nu}-\frac{1}{2} g_{\mu \nu} \square R+2 R_{\mu}^{\alpha} R_{\alpha \nu}-\frac{1}{2} g_{\mu \nu} R^{\alpha \beta} R_{\alpha \beta} \\
& =R_{; \mu \nu}-\frac{1}{2} g_{\mu \nu} \square R-\square R_{\mu \nu}-\frac{1}{2} g_{\mu \nu} R^{\alpha \beta} R_{\alpha \beta}+2 R^{\alpha \beta} R_{\alpha \mu \beta \nu} \\
H_{\mu \nu} & =\frac{1}{\sqrt{-g}} \frac{\delta}{\delta g^{\mu \nu}} \int d^{n} x \sqrt{-g} R^{\alpha \beta \gamma \delta} R_{\alpha \beta \gamma \delta} \\
& =-\frac{1}{2} g_{\mu \nu} R^{\alpha \beta \gamma \delta} R_{\alpha \beta \gamma \delta}+2 R^{\mu \alpha \beta \nu} R_{\nu}^{\alpha \beta \gamma}-4 \square R_{\mu \nu}+2 R_{; \mu \nu} \\
& -4 R_{\mu \alpha} R_{\nu}^{\alpha}+4 R^{\alpha \beta} R_{\alpha \mu \beta \nu} .
\end{aligned}
$$

For the case $n=4$ in conformally flat space-time one has

$$
H_{\mu \nu}={ }^{(2)} H_{\mu \nu}=\frac{1}{3}{ }^{(1)} H_{\mu \nu} .
$$

The explicit expressions for all these tensors in FRW space-time are of course well-known. However, we will use dimensional regularisation, which implies that we have to perform all the computations for general $n=4-\epsilon$. We therefore recall the explicit formulae for the relevant tensors in flat $n$ dimensional FRW geometry in Appendix A.

For the spatially isotropic FRW universe the Einstein field equations reduce for the time-time component and trace to the Friedmann equations

$$
\begin{aligned}
G_{t t} & =-8 \pi G T_{t t}=-8 \pi G \rho \\
G_{\mu}^{\mu} & =-8 \pi G T_{\mu}^{\mu}=-8 \pi G(\rho-(n-1) p),
\end{aligned}
$$

where $\rho$ is the energy density, and the pressure $p$ is given by

$$
p=\left(\rho-T_{\mu}^{\mu}\right) /(n-1) .
$$

. The covariant conservation of energy takes then the form

$$
\frac{d \rho}{d t}=(n-1) H(\rho+p)=H\left(n \rho-T_{\mu}^{\mu}\right) .
$$




\section{Coupled fields in curved space-time}

We here consider the quantum field theory of $N$ coupled scalar fields. More specifically we consider a class of models whose Lagrangian is given by

$$
\mathcal{L}=\sqrt{-g}\left\{\frac{1}{2} \sum_{i} g^{\mu \nu} \partial_{\mu} \Phi_{i} \partial_{\nu} \Phi_{i}-V(\Phi)-W(R, \Phi)\right\},
$$

with the potential

$$
V(\Phi)=\frac{1}{2} \sum_{i=1}^{N} m_{i}^{2} \Phi_{i}^{2}+\frac{1}{4} \sum_{i=1}^{N} \sum_{j=1}^{N} \lambda_{i j} \Phi_{i}^{2} \Phi_{j}^{2} .
$$

Instead $W(R, \phi)$ describes the coupling of the scalar fields to the curvature scalar:

$$
W(R, \Phi)=\sum_{i} \frac{\xi_{i}}{2} R \Phi_{i}^{2}
$$

The $\xi_{i}$ are dimensionless coupling constants that have no counterpart in the flat space theory. As we will see later there is an obviously preferred choice $\xi_{i}=1 / 6$, called conformal coupling. The value $\xi_{i}=0$ is called instead minimal coupling.

\subsection{The equation of motion in the one-loop approxi- mation}

We split the fields in two parts, the expectation values and the quantum fluctuations around it

$$
\Phi_{i}=\phi_{i}(t)+\psi_{i}(t, \boldsymbol{x}),
$$

where the classical field $\phi_{i}(t)$ is assumed to be homogeneous in space. The classical part of the Lagrangian density retains the form

$$
\begin{aligned}
& \mathcal{L}^{(0)}=\sqrt{-g}\left\{\frac{1}{2} \sum_{i}\left[g^{\mu \nu} \partial_{\mu} \phi_{i} \partial_{\nu} \phi_{i}-\left(m_{i}^{2}+\xi_{i} R\right) \phi_{i}^{2}\right]\right. \\
& \left.-\frac{1}{4} \sum_{i j} \lambda_{i j} \phi_{i}^{2} \phi_{j}^{2}-\tilde{\Lambda}\right\}
\end{aligned}
$$

The first order in the fluctuations vanishes (the expectation value of the fluctuations is zero). The fluctuation Lagrangian density of the second order 
in fluctuations

$$
\begin{aligned}
& \mathcal{L}^{(2)}=\sqrt{-g}\left\{\sum_{i} \frac{1}{2} g^{\mu \nu} \partial_{\mu} \psi_{i} \partial_{\nu} \psi_{i}-\frac{1}{2} \sum_{i}\left(m_{i}^{2}+\xi_{i} R\right) \psi_{i}^{2}\right. \\
& \left.-\frac{1}{2} \sum_{i j} \lambda_{i j}\left(\phi_{i}^{2} \psi_{j}^{2}+2 \phi_{i} \phi_{j} \psi_{i} \psi_{j}\right)\right\} \text {. }
\end{aligned}
$$

Now we can write the equations of motion for classical fields:

$$
\begin{aligned}
& \ddot{\phi}_{i}+(n-1) H \dot{\phi}_{i}+\left(m_{i}^{2}+\xi_{i} R\right) \phi_{i} \\
& \quad+\sum_{j} \lambda_{i j}\left[\left(\phi_{j}^{2}+<\psi_{j}^{2}>\right) \phi_{i}+2<\psi_{i} \psi_{j}>\phi_{j}\right]=0,
\end{aligned}
$$

and for the quantum fluctuations in the one-loop approximation:

$$
\ddot{\psi}_{i}+(n-1) H \dot{\psi}_{i}+\frac{1}{a^{2}} \nabla^{2} \psi_{i}+\left(m_{i}^{2}+\xi_{i} R\right) \psi_{i}+\sum_{j} \lambda_{i j}\left(2 \phi_{i} \phi_{j} \psi_{j}+\phi_{j}^{2} \psi_{i}\right)=0 \text {. }
$$

The latter equation can be written in the form

$$
\ddot{\psi}_{i}+(n-1) H \dot{\psi}_{i}+\frac{1}{a^{2}} \nabla^{2} \psi_{i}+\sum_{j} \mathcal{M}_{i j}^{2} \psi_{j}=0,
$$

where the mass squared matrix is given by

$$
\mathcal{M}_{i j}^{2}=\left(m_{i}^{2}+\xi_{i} R\right) \delta_{i j}+2 \lambda_{i j} \phi_{i} \phi_{j}+\delta_{i j} \sum_{k} \lambda_{i k} \phi_{k}^{2}
$$

or, more explicitly,

$$
\begin{aligned}
& \mathcal{M}_{i i}^{2}=m_{i}^{2}+\xi_{i} R+3 \lambda_{i i} \phi_{i}^{2}+\sum_{k \neq i} \lambda_{i k} \phi_{k}^{2}, \\
& \mathcal{M}_{i j}^{2}=2 \lambda_{i j} \phi_{i} \phi_{j} \quad i \neq j .
\end{aligned}
$$

The expectation values of the quantum fluctuations can be expressed in terms of equal-time Green's functions via

$$
<\psi_{i} \psi_{j}>=-i G_{i j}(t, x ; t, x),
$$

where the Green's functions satisfy

$$
\begin{gathered}
\left\{\left[\frac{\partial^{2}}{\partial t^{2}}+(n-1) H \frac{\partial}{\partial t}+\frac{1}{a^{2}} \nabla^{2}\right] \delta_{i j}+\mathcal{M}_{i j}^{2}(t)\right\} G_{j k}\left(t, \boldsymbol{x} ; t^{\prime}, \boldsymbol{x}^{\prime}\right) \\
=\delta_{i k} \frac{i}{a^{n-1}} \delta^{n-1}\left(\boldsymbol{x}-\boldsymbol{x}^{\prime}\right) \delta\left(t-t^{\prime}\right)
\end{gathered}
$$


We have introduced here the set of equations for the classical fields and for the fluctuations in a heuristic way. A rigorous derivation can be found in Ref. [39, based on the Schwinger-Keldysh or closed-time path formalism [40, 41].

The differential operator in Eq. (3.14) is non-Hermitian due to the term $(n-1) H \partial / \partial t$, and so is the matrix valued Green's function $G$. This problem can be solved by introducing conformal time

$$
\tau=\int_{0}^{t} d t^{\prime} \frac{1}{a\left(t^{\prime}\right)}
$$

We can then introduce the dimensionless rescaled fields and their fluctuations

$$
\begin{aligned}
\phi_{i}(t) & =a^{-n / 2+1} \tilde{\phi}_{i}(\tau), \\
\psi_{i}(t, \mathbf{x}) & =a^{-n / 2+1} \tilde{\psi}_{i}(\tau, \mathbf{x}) .
\end{aligned}
$$

as well as the rescaled Green's function

$$
\tilde{G}_{i j}\left(\tau, \mathbf{x} ; \tau^{\prime}, \mathbf{x}^{\prime}\right)=a^{n / 2-1}(t) a^{n / 2-1}\left(t^{\prime}\right) G_{i j}\left(t, \mathbf{x} ; t^{\prime}, \mathbf{x}^{\prime}\right) .
$$

As a function of conformal time, the Hubble parameter, the Ricci scalar and the derivatives of the fields are given by

$$
\begin{aligned}
H & =\frac{a^{\prime}}{a^{2}}, \\
R & =2(n-1) \frac{a^{\prime \prime}}{a^{3}}+(n-1)(n-4) H^{2}, \\
\dot{f} & =a^{-n / 2}\left[\tilde{f}^{\prime}-\frac{n-2}{2} a H \tilde{f}\right], \\
\ddot{f} & =a^{-n / 2-1}\left[\tilde{f}^{\prime \prime}-(n-1) a H \tilde{f}^{\prime}+\frac{(n-1)(n-2)}{2} a^{2} H^{2} \tilde{f}\right. \\
& \left.\quad-a^{2} \frac{n-2}{4(n-1)} R \tilde{f}\right],
\end{aligned}
$$

where $f$ stands for any field and the prime denotes the derivative with respect to conformal time.

Then the equations of motion of the classical fields become

$$
\tilde{\phi}_{i}^{\prime \prime}+\left(m_{i}^{2}+\left(\xi_{i}-\xi_{n}\right) R\right) a^{2} \tilde{\phi}_{i}+a^{4-n} \sum_{j} \lambda_{i j}\left[\left(\phi_{j}^{2}+\tilde{G}_{j j}\right) \phi_{i}+2 \tilde{G}_{i j} \phi_{j}\right]=0,
$$

where

$$
\xi_{n}=\frac{n-2}{4(n-1)}
$$


We see here that for $n=4$ and conformal coupling $\xi_{i}=1 / 6$, the curvature term disappears from the equation of motion of the fields, which reduces to the form it has in flat Minkowski space if also $m_{i}=0$.

The new two-point functions satisfy now

$$
\left[\left(\frac{\partial^{2}}{\partial \tau^{2}}-\nabla^{2}\right) \delta_{i j}+\tilde{\mathcal{M}}_{i j}^{2}(\tau)\right] \tilde{G}_{i j}=-\delta\left(\tau-\tau^{\prime}\right) \delta^{n-1}\left(\mathbf{x}-\mathbf{x}^{\prime}\right),
$$

with the effective masses of the rescaled fluctuation fields

$$
\begin{aligned}
& \tilde{\mathcal{M}}_{i i}^{2}(\tau)=\left(m_{i}^{2}+\left(\xi_{i}-\xi_{n}\right) R\right) a^{2}+a^{4-n}\left(3 \lambda_{i i} \tilde{\phi}_{i}^{2}+\sum_{j \neq i} \lambda_{i j} \tilde{\phi}_{j}^{2}\right) \\
& \tilde{\mathcal{M}}_{i j}^{2}(\tau)=2 a^{4-n} \lambda_{i j} \tilde{\phi}_{i} \tilde{\phi}_{j} \quad i \neq j .
\end{aligned}
$$

The equations for the fluctuation fields in conformal time become

$$
\tilde{\psi}_{i}^{\prime \prime}+\nabla^{2} \tilde{\psi}_{i}+\sum_{j} \tilde{\mathcal{M}}_{i j}^{2}(\tau) \tilde{\psi}_{j}=0 .
$$

We expand the fluctuation fields in terms of mode functions $f_{i}^{\alpha}(\tau ; \boldsymbol{k})$

$$
\tilde{\psi}_{i}(\tau, \boldsymbol{x})=\int \frac{d^{n-1} k}{(2 \pi)^{n-1}} e^{i \boldsymbol{k} \boldsymbol{x}} f_{i}^{\alpha}(\tau ; \boldsymbol{k})
$$

which satisfy the mode equations

$$
f_{i}^{\alpha \prime \prime}(\tau ; \boldsymbol{k})+k^{2} f_{i}^{\alpha}(\tau ; \boldsymbol{k})+\sum_{j} \tilde{\mathcal{M}}_{i j}^{2}(\tau) f_{j}^{\alpha}(\tau ; \boldsymbol{k})=0 .
$$

The latin subscripts refer to the field components, $i=1, \ldots, N$ and the greek superscripts $\alpha=1, \ldots, N$ refer to the $N$ independent solutions of the system of coupled differential equations (3.25). We will specify them below, by a suitable set of initial conditions.

The Green's functions can be expressed by their Fourier components

$$
\tilde{G}_{i j}\left(\tau, \boldsymbol{x} ; \tau^{\prime}, \boldsymbol{x}^{\prime}\right)=\int \frac{d^{n-1} k}{(2 \pi)^{n-1}} e^{i \boldsymbol{k}\left(\boldsymbol{x}-\boldsymbol{x}^{\prime}\right)} \tilde{G}_{i j}\left(\tau, \tau^{\prime} ; \boldsymbol{k}\right) .
$$

These Fourier components can be rewritten in terms of mode functions. They read

$$
\begin{aligned}
\tilde{G}_{i j}\left(\tau, \tau^{\prime} ; \boldsymbol{k}\right)= & \sum_{\alpha \beta} W_{\beta \alpha}^{-1}\left[f_{i}^{\alpha}(\tau, k) f_{j}^{* \beta}\left(\tau^{\prime}, k\right) \theta\left(\tau-\tau^{\prime}\right)\right. \\
& \left.+f_{i}^{\alpha}\left(\tau^{\prime}, k\right) f_{j}^{* \beta}(\tau, k) \theta\left(\tau^{\prime}-\tau\right)\right]
\end{aligned}
$$


where $W_{\alpha \beta}$ is the Wronskian matrix of the system of solutions:

$$
W^{\alpha \beta}=\left[\sum_{j} f_{j}^{\alpha *} f_{j}^{\beta \prime}-f_{j}^{\alpha * \prime} f_{j}^{\beta}\right] .
$$

Therefore the expectation values of the fluctuation fields are given by the fluctuation integrals:

$$
\mathcal{F}_{i j}=<\tilde{\psi}_{i} \tilde{\psi}_{j}>=-i \int \frac{d^{n-1} k}{(2 \pi)^{n-1}} \sum_{\alpha \beta} W_{\beta \alpha}^{-1} \operatorname{Re}\left(f_{i}^{\alpha} f_{j}^{\beta *}\right) .
$$

The Wronskian matrix is determined by the initial conditions of the system of fluctuations which will be discussed in the next subsection.

\subsection{Initial conditions}

The choice of initial conditions for the quantum system is very important for the renormalisation of the equations of motion. In order to satisfy the canonical commutation relations for the creation and annihilation operators and choose a Fock space basis, we first diagonalise the mass matrix $\tilde{\mathcal{M}}_{i j}^{2}$ at the initial time. The eigenvalues $m_{\alpha 0}^{2}, \alpha=1, N$ then define $N$ independent free particle Fock spaces whose product we choose as our initial state. The eigenvalues and eigenvectors of the initial mass matrix $\tilde{\mathcal{M}}_{i j}^{2}(0)$ are defined by the equations

$$
\operatorname{det}\left\{\tilde{\mathcal{M}}_{i j}^{2}(0)-m_{\alpha 0}^{2} \delta_{i j}\right\}=0
$$

and

$$
-m_{\alpha 0}^{2} f_{i 0}^{\alpha}+\tilde{\mathcal{M}}_{i j}^{2}(0) f_{j 0}^{\alpha}=0 .
$$

The eigenvectors $f_{i 0}^{\alpha}$ are orthogonal, we choose them real and normalised as

$$
\sum_{l} f_{l 0}^{\alpha} f_{l 0}^{\beta}=\delta_{\alpha \beta}, \quad \sum_{\alpha} f_{l 0}^{\alpha} f_{m 0}^{\alpha}=\delta_{l m} .
$$

We now specify the mode functions introduced in the previous section by the initial conditions

$$
\begin{aligned}
f_{l}^{\alpha}(0, k) & =f_{l 0}^{\alpha}, \\
f_{l}^{\alpha^{\prime}}(0, k) & =-i \Omega_{\alpha 0} f_{l 0}^{\alpha},
\end{aligned}
$$

with the frequencies $\Omega_{\alpha 0}(0)=\sqrt{k^{2}+m_{\alpha 0}^{2}}$. The Wronskian matrix of the mode functions at time $\tau=0$ is given by

$$
\begin{aligned}
& W_{\alpha \beta}=W\left(f^{\alpha}(0, k), f^{\beta}(0, k)\right) \\
& =\sum_{l}\left[f_{l}^{\alpha *}(0, k) f_{l}^{\beta \prime}(0, k)-f_{l}^{\alpha * \prime}(0, k) f_{l}^{\beta}(0, k)\right]=-2 i \Omega_{\alpha 0} \delta_{\alpha \beta} .
\end{aligned}
$$


It is time-independent by construction. With these initial conditions the fluctuation integrals are given by

$$
\mathcal{F}_{i j}=<\tilde{\psi}_{i} \tilde{\psi}_{j}>=\int \frac{d^{n-1} k}{(2 \pi)^{n-1}} \sum_{\alpha} \frac{1}{2 \Omega_{\alpha 0}} \operatorname{Re}\left(f_{i}^{\alpha} f_{j}^{\alpha *}\right) .
$$

These initial conditions correspond to a quantum state which is denoted as the adiabatic vacuum. For such an initial state the energy-momentum tensor is found to be singular at initial time [42]. This problem can be solved by a suitable Bogoliubov transformations of the adiabatic vacuum. For a coupled system this Bogoliubov transformation was derived in Ref. [43]. We recall the essential features of this approach in Appendix B. As discussed in Ref. [42] this modification of the initial state does not affect the renormalisation, which is our main subject here; so we continue to work with the adiabatic vacuum as the initial state.

\subsection{The energy-momentum tensor}

In order to write the Einstein's equations we need the expression of the energy-momentum tensor for our system of coupled field in Eq. (3.1). Generalising straightforwardly the case of a single scalar field given in Ref. [38], we have

$$
\begin{aligned}
T_{\mu \nu}= & \sum_{i}\left[\left(1-2 \xi_{i}\right) \Phi_{i ; \mu} \Phi_{i ; \nu}+\left(2 \xi_{i}-\frac{1}{2}\right) g_{\mu \nu} g^{\rho \sigma} \Phi_{i ; \rho} \Phi_{i ; \sigma}-2 \xi_{i} \Phi_{i ; \mu \nu} \Phi_{i}\right. \\
& \left.+2 \xi_{i} g_{\mu \nu} \Phi_{i} \square \Phi_{i}-\xi_{i} G_{\mu \nu} \Phi_{i}^{2}\right]+g_{\mu \nu} V(\Phi) .
\end{aligned}
$$

In the Friedmann equations only $T_{t t}$ and $T_{\mu}^{\mu}$ appear and their classical parts are given by

$$
\begin{aligned}
T_{t t}^{\mathrm{cl}}= & \sum_{i}\left(\frac{1}{2} \dot{\phi}_{i}{ }^{2}-\xi_{i} G_{t t} \phi_{i}^{2}+2(n-1) \xi_{i} H \phi_{i} \dot{\phi}_{i}\right)+V^{c l}(\phi) \\
T_{\mu}^{\mathrm{cl} \mu}= & \sum_{i}\left\{\left[1-\frac{n}{2}+2(n-1) \xi_{i}\right] \dot{\phi}_{i}{ }^{2}+2(n-1) \xi_{i}\left[\ddot{\phi}_{i}+(n-1) H \dot{\phi}_{i}\right] \phi_{i}\right. \\
& \left.-\xi_{i} G_{\mu}^{\mu} \phi_{i}^{2}\right\}+n V^{c l}(\phi)
\end{aligned}
$$

with

$$
V^{\mathrm{cl}}(\phi)=\frac{1}{2} \sum_{i} m_{i}^{2} \phi_{i}^{2}+\frac{1}{4} \sum_{i, j} \lambda_{i j} \phi_{i}^{2} \phi_{j}^{2}
$$


Using conformal time and the rescaled fields we find 3

$$
\begin{aligned}
T_{t t}^{\mathrm{cl}} & =\frac{1}{a^{n}}\left\{\frac{1}{2} \sum_{i}\left(\tilde{\phi}_{i}^{\prime 2}+m_{i}^{2} a^{2} \tilde{\phi}_{i}^{2}\right)\right. \\
& +2(n-1) \sum_{i}\left(\xi_{i}-\xi_{n}\right)\left(a H \tilde{\phi}_{i}^{\prime}-\frac{n-2}{4} a^{2} H^{2} \tilde{\phi}_{i}\right) \tilde{\phi}_{i} \\
& \left.+\frac{a^{4-n}}{4} \sum_{i j} \lambda_{i j} \tilde{\phi}_{i}^{2} \tilde{\phi}_{j}^{2}\right\} .
\end{aligned}
$$

For the fluctuation contribution to the energy density we obtain instead

$$
\begin{aligned}
& T_{t t}^{\mathrm{q}}=\frac{1}{a^{n}} \int \frac{d^{n-1} k}{(2 \pi)^{n-1}} \sum_{\alpha} \frac{1}{2 \Omega_{\alpha 0}} \\
& \times\left\{\frac{1}{2} \sum_{i}\left[\left|f_{i}^{\alpha \prime}\right|^{2}+k^{2}\left|f_{i}^{\alpha}\right|^{2}\right]+\frac{1}{2} \sum_{i j} \tilde{\mathcal{M}}_{i j}^{2}(\tau) f_{i}^{\alpha *} f_{j}^{\alpha}\right. \\
& \left.+(n-1) \sum_{i}\left(\xi_{i}-\xi_{n}\right)\left[a H \frac{d}{d \tau}\left|f_{i}^{\alpha}\right|^{2}-\left(\frac{n-2}{2} a^{2} H^{2}+\frac{R a^{2}}{2(n-1)}\right)\left|f_{i}^{\alpha}\right|^{2}\right]\right\} .
\end{aligned}
$$

The last term, proportional to $R$, which has no analogy in the classical energy-momentum tensor, is not genuine but compensates an analogous term in $\tilde{\mathcal{M}}_{i j}^{2}$. It is convenient to rewrite $T_{t t}^{\mathrm{q}}$ as a function of the fluctuation integral as

$$
\begin{aligned}
T_{t t}^{\mathrm{q}}= & T_{t t}^{\mathrm{q}, \mathrm{kin}}+\frac{1}{2 a^{n}} \mathcal{V}_{i j} \mathcal{F}_{i j} \\
& +\frac{n-1}{a^{n}} \sum_{i}\left(\xi_{i}-\xi_{n}\right)\left[a H \frac{d}{d \tau} \mathcal{F}_{i i}-\left(\frac{n-2}{2} a^{2} H^{2}+\frac{R a^{2}}{2(n-1)}\right) \mathcal{F}_{i i}\right],
\end{aligned}
$$

where we have introduced the "potential"

$$
\mathcal{V}_{i j}(\tau)=\tilde{\mathcal{M}}_{i j}^{2}(\tau)-\tilde{\mathcal{M}}_{i j}^{2}(0)
$$

and where

$$
\begin{aligned}
T_{t t}^{\mathrm{q}, \mathrm{kin}}= & \frac{1}{a^{n}} \int \frac{d^{n-1} k}{(2 \pi)^{n-1}} \sum_{\alpha} \frac{1}{2 \Omega_{\alpha 0}}\left\{\frac{1}{2} \sum_{i}\left[\left|f_{i}^{\alpha^{\prime}}\right|^{2}+\Omega_{\alpha 0}^{2}\left|f_{i}^{\alpha}\right|^{2}\right]\right. \\
& \left.+\frac{1}{2} \sum_{i j}\left(\tilde{\mathcal{M}}_{i j}^{2}(0)-m_{\alpha 0}^{2} \delta_{i j}\right) f_{i}^{\alpha *} f_{j}^{\alpha}\right\}
\end{aligned}
$$

\footnotetext{
${ }^{3}$ Though we have introduced conformal time we continue to use the time-time component of the energy-momentum tensor in standard time $t$, as made explicit by using time indices $t$ instead of 0 or $\tau$.
} 
is the kinetic or free-field part of the energy density.

The classical part of the trace of the energy-momentum tensor becomes

$$
\begin{aligned}
T_{\mu}^{\mathrm{cl} \mu}= & \frac{1}{a^{n}}\left\{2(n-1) \sum_{i}\left(\xi_{i}-\xi_{n}\right)\left[\tilde{\phi}_{i}^{\prime}-\frac{1}{2}(n-2) a H \tilde{\phi}_{i}\right]^{2}\right. \\
& \left.+2(n-1) \sum_{i} \xi_{i} \tilde{\phi}_{i} \tilde{\phi}_{i}^{\prime \prime}+n\left[\frac{1}{2} \sum_{i} m_{i}^{2} \tilde{\phi}_{i}^{2}+\frac{1}{4} \sum_{i j} \lambda_{i j} a^{4-n} \tilde{\phi}_{i}^{2} \tilde{\phi}_{j}^{2}+\Lambda\right]\right\} .
\end{aligned}
$$

For the quantum contribution one finds instead

$$
\begin{aligned}
T_{\mu}^{\mathrm{q} \mu}= & \frac{1}{a^{n}} \int \frac{d^{n-1} k}{(2 \pi)^{n-1}} \sum_{\alpha} \frac{1}{2 \Omega_{\alpha 0}} \\
& \times\left\{2 ( n - 1 ) \sum _ { i } ( \xi _ { i } - \xi _ { n } ) \left[\left|f_{i}^{\alpha \prime}\right|^{2}-k^{2}\left|f_{i}^{\alpha}\right|^{2}-\mathcal{M}_{i j}^{2} f_{i}^{\alpha *} f_{j}^{\alpha}\right.\right. \\
& \left.-\frac{n-2}{2} a H \frac{d}{d \tau}\left|f_{i}^{\alpha}\right|^{2}+\frac{1}{4}\left((n-2)^{2} H^{2}-\frac{n}{n-1} R\right)\left|f_{i}^{\alpha}\right|^{2}\right] \\
& \left.+\sum_{i j} \mathcal{M}_{i j}^{2} f_{i}^{\alpha *} f_{j}^{\alpha}\right\} .
\end{aligned}
$$

Using the equation of motion for the mode functions, Eq. (3.25), the first three terms in the bracket can we rewritten as

$$
\left|f_{i}^{\alpha \prime}\right|^{2}-k^{2}\left|f_{i}^{\alpha}\right|^{2}-\mathcal{M}_{i j}^{2} f_{i}^{\alpha *} f_{j}^{\alpha}=\left|f_{i}^{\alpha^{\prime}}\right|^{2}+f_{i}^{\alpha^{*}} f_{i}^{\alpha \prime \prime}=\frac{1}{2} \frac{d^{2}}{d \tau^{2}}\left|f_{i}^{\alpha}\right|^{2} .
$$

We therefore may express the entire quantum contribution to $T_{\mu}^{\mu}$ in terms of the fluctuations integral $\mathcal{F}_{i j}$ as

$$
\begin{aligned}
T_{\mu}^{\mathrm{q} \mu}= & \frac{n-1}{a^{n}} \sum_{i}\left(\xi_{i}-\xi_{n}\right)\left[\frac{d^{2}}{d \tau^{2}} \mathcal{F}_{i i}-(n-2) a H \frac{d}{d \tau} \mathcal{F}_{i i}\right. \\
& \left.+\frac{1}{2}\left((n-2)^{2} H^{2}-\frac{n}{n-1} R\right) \mathcal{F}_{i i}\right]+\frac{1}{a^{n}} \sum_{i j} \mathcal{M}_{i j}^{2} \mathcal{F}_{i j} .
\end{aligned}
$$

\section{Renormalisation}

In the previous sections we have introduced the fluctuation integrals and expressed the equations of motion and the one-loop contributions to the energy-momentum tensor in term of these quantities. These definitions have 
only formal character, as they involve divergent momentum integrals. As in the case of renormalisation in Minkowski, we have to introduce regulators in order to render the integrals well-defined, and counterterms to absorb the divergences when the regulators are removed. We will use here dimensional regularisation with the space dimension $3-\epsilon$. Furthermore, it is convenient to separate the limit $\epsilon \rightarrow 0$ entirely from the numerical computations by adding and subtracting appropriate analytical terms, which reproduce the divergent behaviour and render the numerical integrations finite. Such a scheme was set up in Ref. [37, and we will use it here. We recall its main features and the formulae needed here in Appendices C, D and E.

Before we discuss renormalisation we have to discuss a subtle issue (see also Ref. [36]). We have written all equations for general $n=4-\epsilon$, and the equations of motion ensure that the energy-momentum tensor is conserved in $n$ dimensions. Ultimately we have to take the limit $n \rightarrow 4$, or $\epsilon \rightarrow 0$ and this will generate terms that behave as $1 /(n-4)$ from the divergent integrals and consequently in the counterterm action. On the other hand the equations of motion and the energy-momentum tensor are equal to their 4dimensional form only up to terms of order $(n-4)$. These terms will survive the limit $n \rightarrow 4$ whenever they are multiplied by a factor $1 /(n-4)$. This will result in "additional finite terms", which have no counterpart in quantum field theory in Minkowski space. So our prescription is: we first formulate the full renormalised theory in $n$ dimensions and take the limit $n \rightarrow 4$ only at the end. Another prescription one may think to use, is consider $n \neq 4$ only in the divergent integrals, while keeping the equation of motion and the energy-momentum tensor in 4 dimensions, but in that case we cannot rely on energy-momentum covariant conservation during the whole regularisation procedure.

\subsection{Renormalisation of the equation of motion}

The equation of motion contains logarithmic and quadratic divergences due to the fluctuation integrals. Using the analysis of Appendix $\mathrm{C}$ and in particular Eq. (C.12), their divergent behaviour can be understood and it coincides with that of the divergent integral

$$
\mathcal{F}_{i j}^{d i v}=a^{\epsilon} \int \frac{d^{n-1} k}{(2 \pi)^{n-1}} \sum_{\alpha} \frac{1}{2 \Omega_{\alpha 0}}\left[f_{i 0}^{\alpha} f_{j 0}^{\alpha}-\sum_{\beta} f_{i 0}^{\alpha} f_{j 0}^{\beta} \frac{\tilde{\mathcal{V}}_{\alpha \beta}(\tau)}{\Omega_{\beta 0}\left(\Omega_{\alpha 0}+\Omega_{\beta 0}\right)}\right],
$$

where the potential $\tilde{\mathcal{V}}_{\alpha \beta}$ has been introduced in Eq. (C.10) Here and in the following we use a slight change in notation, without introducing a new sym- 
bol: we incorporate the prefactor $a^{\epsilon}$ which appears in front of the divergent integrals into the $n$-dimensional integration measure.

Using the formulae for the various integrals given in Appendix E one can obtain analytically the dimensionally regulated form of the previous expression as

$$
\begin{aligned}
\mathcal{F}_{i j}^{\mathrm{reg}}= & -\frac{L_{\epsilon}}{16 \pi^{2}} \tilde{\mathcal{M}}_{i j}^{2}(\tau)+\sum_{\alpha} \frac{m_{\alpha 0}^{2}}{16 \pi^{2}} f_{i 0}^{\alpha} f_{j 0}^{\alpha}\left(\ln \frac{m_{\alpha 0}^{2}}{a^{2} \mu^{2}}-1\right) \\
& +\sum_{\alpha, \beta} f_{i 0}^{\alpha} f_{j 0}^{\beta} \frac{\tilde{\mathcal{V}}_{\alpha \beta}(\tau)}{16 \pi^{2}}\left(\ln \frac{m_{\alpha 0}^{2}}{a^{2} \mu^{2}}-\frac{m_{\beta 0}^{2}}{m_{\alpha 0}^{2}-m_{\beta 0}^{2}} \ln \frac{m_{\beta 0}^{2}}{m_{\alpha 0}^{2}}-1\right),
\end{aligned}
$$

where $L_{\epsilon}=\frac{2}{\epsilon}-\gamma+\ln 4 \pi$. In order to cancel this divergence in the equations of motion we have to add an appropriate counterterm to the Lagrange density. In the case of Minkowski space-time, it was found in Ref. [27] that the counterterm Lagrangian

$$
\mathcal{L}_{\text {Mink. }}^{\mathrm{ct}}=-\delta \zeta \mathcal{M}_{k l}^{2} \mathcal{M}_{l k}^{2}
$$

with

$$
\delta \zeta=\frac{L_{\epsilon}}{64 \pi^{2}}
$$

takes account of all divergences. We work here in the $\overline{M S}$ scheme and subtract only the divergent part of the fluctuation integral. Note that for constant masses and fields, this is a contribution to the renormalisation of the cosmological constant of order $\sum_{i} m_{i}^{4}$ and also contains mass and coupling renormalisation terms due to the interaction part of the effective mass matrix $\mathcal{M}_{i j}^{2}$.

Taking into account the factor $\sqrt{-g}=a^{n}$ (using conformal time), and noting that the $\tilde{\mathcal{M}}_{i j}^{2}$ differ by a factor $a^{2}$ from $\mathcal{M}_{i j}^{2}$, the equivalent Lagrangian for general FRW is

$$
\mathcal{L}^{\mathrm{ct}}=-a^{n-4} \delta \zeta \tilde{\mathcal{M}}_{k l}^{2} \tilde{\mathcal{M}}_{l k}^{2}
$$

When discussing the energy-momentum tensor it will be convenient to have this Lagrangian decomposed into the usual mass, coupling constant and other gravity-related counterterms. This is presented in Appendix $\mathrm{F}$.

Introducing this counterterm Lagrangian and given that

$$
\begin{aligned}
\frac{\partial}{\partial \tilde{\phi}_{i}} \mathcal{L}^{\mathrm{ct}} & =2 \delta \zeta \tilde{\mathcal{M}}_{k l}^{2}\left(4 \lambda_{i l} \tilde{\phi}_{l} \delta_{i k}+2 \delta_{k l} \lambda_{k i} \tilde{\phi}_{i}\right) \\
& =4 \delta \zeta \sum_{l}\left[2 \tilde{\mathcal{M}}_{i l}^{2} \lambda_{i l} \tilde{\phi}_{l}+\lambda_{l i} \tilde{\mathcal{M}}_{l l}^{2} \tilde{\phi}_{i}\right]
\end{aligned}
$$


the equations of motion for the classical fields become

$$
\begin{aligned}
& \tilde{\phi}_{i}^{\prime \prime}+\left(m_{i}^{2}+\left(\xi_{i}-\frac{1}{6}\right) R\right) a^{2} \tilde{\phi}_{i} \\
& +\sum_{j} \lambda_{i j}\left[\left(\phi_{j}^{2}+4 \delta \zeta \tilde{\mathcal{M}}_{j j}^{2}+\mathcal{F}_{j j}\right) \tilde{\phi}_{i}+\left(8 \delta \zeta \tilde{\mathcal{M}}_{i j}^{2}+2 \mathcal{F}_{i j}\right) \tilde{\phi}_{j}\right]=0 .
\end{aligned}
$$

It follows from Eq. (4.2) and the renormalisation procedure that the combination $4 \delta \zeta \tilde{\mathcal{M}}_{i j}^{2}+\mathcal{F}_{i j}$ is finite. This is entirely analogous to the case without coupling to gravity. There is, however, one important difference: in addition to the renormalisation scale the scale factor $a(\tau)$ appears in the prefactor $a^{\epsilon}$ and this modifies the finite terms. In order to be able to identify the different contributions to $\mathcal{F}_{i j}$ separately we write

$$
\mathcal{F}_{i j}=-\frac{L_{\epsilon}}{16 \pi^{2}} \tilde{\mathcal{M}}_{i j}^{2}(\tau)+\mathcal{F}_{i j}^{\mathrm{fin}}+\mathcal{F}_{i j}^{\mathrm{add}},
$$

where the finite part of the fluctuation integral is given by

$$
\mathcal{F}_{i j}^{\mathrm{fin}}=\mathcal{F}_{i j}^{\mathrm{sub}}+\mathcal{F}_{i j}^{\mathrm{ft}}
$$

Here we define

$$
\mathcal{F}_{i j}^{\mathrm{sub}}=\int \frac{k^{2} d k}{2 \pi^{2}} \sum_{\alpha} \frac{1}{2 \Omega_{\alpha 0}}\left[\operatorname{Re}\left(f_{i}^{\alpha} f_{j}^{\alpha *}\right)-f_{i 0}^{\alpha} f_{j 0}^{\alpha}+\sum_{\beta} f_{i 0}^{\alpha} f_{j 0}^{\beta} \frac{\tilde{\mathcal{V}}_{\alpha \beta}(\tau)}{\Omega_{\beta 0}\left(\Omega_{\alpha 0}+\Omega_{\beta 0}\right)}\right]
$$

as the subtracted fluctuation integral, that is finite, but in general has to be computed numerically. From $\mathcal{F}_{i j}^{\text {reg }}$, Eq. (4.2), we have

$$
\begin{aligned}
\mathcal{F}_{i j}^{\mathrm{ft}}= & \sum_{\alpha}\left[\frac{m_{\alpha 0}^{2}}{16 \pi^{2}} f_{i 0}^{\alpha} f_{j 0}^{\alpha}\left(\ln \frac{m_{\alpha 0}^{2}}{a^{2} \mu^{2}}-1\right)\right. \\
& \left.+\sum_{\beta} f_{i 0}^{\alpha} f_{j 0}^{\beta} \frac{\tilde{\mathcal{V}}_{\alpha \beta}(\tau)}{16 \pi^{2}}\left(\ln \frac{m_{\alpha 0}^{2}}{a^{2} \mu^{2}}-\frac{m_{\beta 0}^{2}}{m_{\alpha 0}^{2}-m_{\beta 0}^{2}} \ln \frac{m_{\beta 0}^{2}}{m_{\alpha 0}^{2}}-1\right)\right]
\end{aligned}
$$

as finite terms that are left over after removing the divergent part. However, among those we have identified separately

$$
\mathcal{F}_{i j}^{\text {add }}=-\frac{1}{288 \pi^{2}} a^{2} R \delta_{i j},
$$

which arises from the $\mathcal{O}(\epsilon)$ term in the expansion of $\tilde{\mathcal{M}}_{i i}^{2}$ :

$\tilde{\mathcal{M}}_{i i}^{2}=\left(m_{i}^{2}+\left(\xi_{i}-1 / 6\right) R\right) a^{2}+a^{4-n}\left(3 \lambda_{i i} \tilde{\phi}_{i}^{2}+\sum_{j \neq i} \lambda_{i j} \tilde{\phi}_{j}^{2}\right)+\frac{\epsilon}{36} a^{2} R+O\left(\epsilon^{2}\right)$. 
This is the only term that has no usual $4 D$ counterpart and is generated from taking the $n$-dimensional FRW model. It gives a finite contribution to the scalar field coupling to gravity $\xi_{i}$ and it may be in principle absorbed into a more general renormalisation scheme than $\overline{M S}$. Note that such term survives even in the case of $4 \mathrm{D}$ conformal coupling $\xi_{i}=1 / 6$.

Finally, the renormalised equation of motion for the classical fields reads

$$
\begin{aligned}
& \tilde{\phi}_{i}^{\prime \prime}+\left(m_{i}^{2}+\left(\xi_{i}-\frac{1}{6}\right) R\right) a^{2} \tilde{\phi}_{i} \\
& +\sum_{j} \lambda_{i j}\left[\left(\tilde{\phi}_{j}^{2}+\mathcal{F}_{j j}^{\mathrm{fin}}+\mathcal{F}_{j j}^{\mathrm{add}}\right) \tilde{\phi}_{i}+2\left(\mathcal{F}_{i j}^{\mathrm{fin}}+\mathcal{F}_{i j}^{\mathrm{add}}\right) \tilde{\phi}_{j}\right]=0 .
\end{aligned}
$$

Here we have taken the limit $\epsilon \rightarrow 0$ already, and it is understood that the potential $\tilde{\mathcal{V}}_{\alpha \beta}$ in $\mathcal{F}_{i j}^{\text {fin }}$ is computed in 4 dimensions. Likewise, the fluctuations are computed using the 4 -dimensional reduction of $\tilde{\mathcal{M}}_{i j}^{2}$ in their equation of motion.

Note that the occurrence of $\ln a(\tau)$ in the finite terms $\tilde{\mathcal{M}}_{i j}^{2}$ is not necessarily a small correction. Neither is the "potential" $\mathcal{V}_{\alpha \beta}$ small: the terms $\left(m_{i}^{2}+\left(\xi_{i}-1 / 6\right) R\right)$ appear multiplied by $a^{2}$. Indeed the qualitative rôle of the $\ln a(\tau)$ term in $\mathcal{F}_{i j}^{\mathrm{ft}}$ is to compensate a logarithmic increase of the subtracted integral $\mathcal{F}_{i j}^{\text {sub }}$. This is illustrated by an example in Appendix G.

\subsection{Renormalisation of the energy-momentum tensor}

We can now proceed in the same way to the renormalisation of the energymomentum tensor. In Sec. 3.3 we have separated the quantum part of the $t t$ component of the energy-momentum tensor into a "kinetic" part and some further contributions which can be written in terms of the fluctuation integrals. The divergences of the latter have been discussed in the previous section, so it remains here to analyse the kinetic part. Using the results of Appendix $\mathrm{C}$ and in particular Eq. (C.18) we obtain

$$
\begin{aligned}
T_{t t}^{\mathrm{q}, \mathrm{kin}}= & \frac{1}{a^{n}} \int \frac{d^{n-1} k}{(2 \pi)^{n-1}} \sum_{\alpha} \frac{1}{2 \Omega_{\alpha 0}}\left[\Omega_{\alpha 0}^{2}+\frac{1}{2}\left|h_{i}^{\alpha \prime}\right|^{2}\right. \\
& \left.+\frac{1}{2}\left(\tilde{\mathcal{M}}_{i j}^{2}(0)-m_{\alpha 0}^{2} \delta_{i j}\right) \operatorname{Re}\left(h_{i}^{\alpha} h_{j}^{\alpha *}\right)\right] .
\end{aligned}
$$

The divergent behaviour of the first term in the bracket is obvious, the one of the second term is given by Eq. (C.20). The third term originally appears with the mode functions $f_{i}^{\alpha}$; the present form, with the reduce mode functions $h_{i}^{\alpha}$, is obtained by using that the parenthesis $\left(\tilde{\mathcal{M}}_{i j}^{2}(0)-m_{\alpha 0}^{2} \delta_{i j}\right)$ vanishes when 
multiplied with $f_{i 0}^{\alpha}$ or $f_{j 0}^{\alpha}$ due to the eigenvalue equation (3.31). Still the term could lead to a divergence arising from the second order contribution $h_{i}^{(1) \alpha} h_{j}^{(1) \alpha *}$. One can convince oneself that this leading term vanishes. The expansion of $h_{i}^{(1) \alpha}$ and $h_{j}^{(1) \alpha *}$ contains factors $f_{i 0}^{\beta}$ or $f_{j 0}^{\beta}$ (see Eqs. (C.8) and (C.9) ); the parenthesis then reduces to $\left(m_{\beta 0}^{2}-m_{\alpha 0}^{2}\right) \delta_{i j}$ and this is found to be multiplied with a term that is symmetric in $\alpha$ and $\beta$. So, using the integrals given in Appendix E we can write

$$
T_{t t}^{\mathrm{q}, \mathrm{kin}}=-\frac{1}{64 \pi^{2} a^{4}} L_{\epsilon}\left[\sum_{\alpha} m_{\alpha_{0}}^{4}-\sum_{\alpha \beta} \tilde{\mathcal{V}}_{\alpha \beta} \tilde{\mathcal{V}}_{\beta \alpha}\right]+T_{t t}^{\mathrm{q}, \mathrm{kin}, \mathrm{fin}}
$$

Here

$$
T_{t t}^{\mathrm{q}, \mathrm{kin}, \mathrm{fin}}=T_{t t}^{\mathrm{q}, \mathrm{kin}, \mathrm{sub}}+T_{t t}^{\mathrm{q}, \mathrm{kin}, \mathrm{ft}},
$$

where the subtracted integral is given by

$$
\begin{aligned}
T_{t t}^{\mathrm{q}, \text { kin,sub }}= & \frac{1}{a^{4}} \int \frac{k^{2} d k}{2 \pi^{2}} \sum_{\alpha} \frac{1}{2 \Omega_{\alpha 0}}\left[\frac{1}{2}\left|h_{i}^{\alpha \prime}\right|^{2}-\sum_{\beta} \frac{\tilde{\mathcal{V}}_{\beta \alpha} \tilde{\mathcal{V}}_{\alpha \beta}}{4 \Omega_{\beta 0}\left(\Omega_{\alpha 0}+\Omega_{\beta 0}\right)}\right. \\
& \left.+\frac{1}{2}\left(\tilde{\mathcal{M}}_{i j}^{2}(0)-m_{\alpha 0}^{2} \delta_{i j}\right) \operatorname{Re}\left(h_{i}^{\alpha} h_{j}^{\alpha *}\right)\right]
\end{aligned}
$$

and the finite terms of the regularised integrals are

$$
\begin{aligned}
& T_{t t}^{\mathrm{q}, \mathrm{kin}, \mathrm{ft}}=\frac{1}{64 \pi^{2} a^{4}}\left[\sum_{\alpha} m_{\alpha_{0}}^{4}\left(\ln \frac{m_{\alpha 0}^{2}}{a^{2} \mu^{2}}-\frac{3}{2}\right)\right. \\
& \left.-\sum_{\alpha \beta} \tilde{\mathcal{V}}_{\alpha \beta} \tilde{\mathcal{V}}_{\beta \alpha}\left(\ln \frac{m_{\alpha 0}^{2}}{a^{2} \mu^{2}}-\frac{m_{\beta 0}^{2}}{m_{\alpha 0}^{2}-m_{\beta 0}^{2}} \ln \frac{m_{\beta 0}^{2}}{m_{\alpha 0}^{2}}-1\right)\right] .
\end{aligned}
$$

In both of these equations it is understood that $n=4$.

Besides $T_{t t}^{\mathrm{q}, \mathrm{kin}}$ the $t t$ component of the energy-momentum tensor contains further quantum contributions, which have been expressed by fluctuation integrals, see Eq. (3.43). Collecting all these finite pieces we define

$$
\begin{aligned}
& T_{t t}^{\mathrm{q}, \mathrm{fin}}=T_{t t}^{\mathrm{q}, \mathrm{kin}, \mathrm{fin}}+\frac{1}{2} \mathcal{V}_{i j} \mathcal{F}_{i j}^{\mathrm{fin}} \\
& +\frac{3}{a^{4}} \sum_{i}\left(\xi_{i}-\frac{1}{6}\right)\left[a H \frac{d}{d \tau} \mathcal{F}_{i i}^{\mathrm{fin}}-\left(a^{2} H^{2}+\frac{R a^{2}}{6}\right) \mathcal{F}_{i i}^{\mathrm{fin}}\right],
\end{aligned}
$$

where we have already set $n=4$. 
For the singular terms we find instead

$$
\begin{aligned}
T_{t t}^{\mathrm{q}, \text { sing }}= & -\frac{L_{\epsilon}}{64 \pi^{2}} \frac{1}{a^{n}} \tilde{\mathcal{M}}_{i j}^{2} \tilde{\mathcal{M}}_{i j}^{2} \\
& +\frac{n-1}{a^{n}} \sum_{i}\left(\xi_{i}-\xi_{n}\right)\left(\frac{n-2}{2} a^{2} H^{2}+\frac{R a^{2}}{2(n-1)}\right) \frac{L_{\epsilon}}{16 \pi^{2}} \tilde{\mathcal{M}}_{i i}^{2} \\
& -\frac{n-1}{a^{n}} \sum_{i}\left(\xi_{i}-\xi_{n}\right) \frac{L_{\epsilon}}{16 \pi^{2}} \frac{d}{d \tau} \tilde{\mathcal{M}}_{i i}^{2}
\end{aligned}
$$

Here it is understood that everything is still defined for general $n$. The counterterms are (see Eqs. (2.6) and (3.41)

$$
\begin{aligned}
& T_{t t}^{\mathrm{ct}}=\frac{1}{a^{n}}\left\{\frac{1}{2} \sum_{i} \delta m_{i}^{2} a^{2} \tilde{\phi}_{i}^{2}\right. \\
& +2(n-1) \sum_{i} \delta \xi_{i}\left(a H \tilde{\phi}_{i}^{\prime}-\frac{n-2}{4} a^{2} H^{2} \tilde{\phi}_{i}\right) \tilde{\phi}_{i} \\
& \left.+\frac{a^{4-n}}{4} \sum_{i j} \delta \lambda_{i j} \tilde{\phi}_{i}^{2} \tilde{\phi}_{j}^{2}\right\}+\delta \tilde{\Lambda}+\delta \tilde{Z} G_{t t}+\delta \tilde{\alpha}^{(1)} H_{t t} .
\end{aligned}
$$

Adding the singular part and the counterterms one finds that the divergences are cancelled, but, as in the case of $\mathcal{F}_{i j}$ some additional finite terms remain in the limit $n \rightarrow 4$. These are

$$
\begin{aligned}
& T_{t t}^{\mathrm{q}, \text { add }}=\lim _{n \rightarrow 4}\left(T_{t t}^{\mathrm{q}, \mathrm{sing}}+T_{t t}^{\mathrm{ct}}\right)=\frac{H}{96 \pi^{2} a^{3}} \sum_{i}\left(a H \tilde{\mathcal{M}}_{i i}^{2}-\frac{d}{d \tau} \tilde{\mathcal{M}}_{i i}^{2}\right) \\
& +\frac{1}{16 \pi^{2}} \sum_{i}\left(\xi_{i}-\frac{1}{6}\right)\left[\frac{1}{36}{ }^{(1)} H_{t t}+\frac{1}{72} R^{2}-6 \frac{H^{2}}{a^{2}} \tilde{\mathcal{M}}_{i i}^{2}\right] .
\end{aligned}
$$

It is understood that $n$ is set equal to 4 in $\tilde{\mathcal{M}}_{i i}^{2}$ and ${ }^{(1)} H_{t t}$. The result agrees with the one for the single-field case found in Ref. [36]. There $T_{t t}^{\mathrm{q}, \text { add }}$ was in addition presented in an expanded form, using the explicit expressions for $\tilde{\mathcal{M}}_{i i}^{2}$ and ${ }^{(1)} H_{t t}$. Here we refrain from displaying such a rather lengthy formula. We should mention that the calculations for checking the cancellation of divergences and for obtaining $T_{t t}^{\mathrm{q}, \text { add }}$ are quite cumbersome, they have been performed using the computer algebra code REDUCE [44]. Note that these additional terms are time-dependent and similar time-dependent pieces are contained in the subtracted integrals eq. (4.18), as it happens for the $\ln a(\tau)$ terms in $\tilde{\mathcal{F}}_{i j}$. Both together ensure that the energy-momentum tensor is covariantly conserved. 
There is still a further finite contribution to the energy-momentum tensor, the conformal anomaly, which can in any case not be renormalised away [38. As discussed there and in Ref. [36] it cannot be determined within our framework but has to be taken over from a more general analysis. It is obtained by setting, for $N$ scalar fields,

$$
\delta \tilde{\beta}=-\delta \tilde{\gamma}=\frac{N}{2880 \pi^{2}} L_{\epsilon} .
$$

As ${ }^{(2)} H_{\mu \nu}=H_{\mu \nu}$ in 4 dimensions, the $n=4$ part of these tensors cancels, and so does the singularity. But there is a finite remainder from the extension to $n \neq 4$. We obtain 36 ]

$$
\begin{aligned}
T_{t t}^{\mathrm{ano}} & =\lim _{n \rightarrow 4} \frac{N}{2880 \pi^{2}} L_{\epsilon}\left({ }^{(2)} H_{t t}-H_{t t}\right) \\
& =\frac{N}{2880 \pi^{2}}\left(H \frac{R^{\prime}}{a}+R H^{2}+\frac{1}{12} R^{2}+3 H^{4}\right) .
\end{aligned}
$$

Notice that these additional terms are vanishing for the case of pure de Sitter or radiation dominated expansion, so they are negligible in the inflationary phase, but they could have an effect in the reheating phase.

Finally, the renormalised $t t$ component of the energy-momentum tensor is given by

$$
T_{t t}^{\mathrm{ren}}=T_{t t}^{\mathrm{cl}}+T_{t t}^{\mathrm{q}, \mathrm{fin}}+T_{t t}^{\mathrm{q}, \text { add }}+T_{t t}^{\mathrm{ano}} .
$$

As we have seen in Sec. 3.3, $T_{\mu}^{\mathrm{q} \mu}$, the unrenormalised quantum contribution to the trace of the energy-momentum tensor can entirely be expressed in terms of the fluctuation integrals $\mathcal{F}_{i j}$ and derivatives thereof, see Eq. (3.50). So the finite part is simply given by

$$
\begin{aligned}
T_{\mu}^{\mathrm{q}, \mathrm{fin} \mu}= & \frac{3}{a^{4}} \sum_{i}\left(\xi_{i}-\frac{1}{6}\right)\left[\frac{d^{2}}{d \tau^{2}} \mathcal{F}_{i i}^{\mathrm{fin}}-2 a H \frac{d}{d \tau} \mathcal{F}_{i i}^{\mathrm{fin}}\right. \\
& \left.+\left(2 H^{2}-\frac{2}{3} R\right) \mathcal{F}_{i i}^{\mathrm{fin}}\right]+\frac{1}{a^{4}} \sum_{i j} \tilde{\mathcal{M}}_{i j}^{2} \mathcal{F}_{i j}^{\mathrm{fin}} .
\end{aligned}
$$

Likewise, the divergences of $T_{\mu}^{\mathrm{q} \mu}$ are related in a straightforward way to those of the fluctuation integrals. Therefore, the part that is singular as $n \rightarrow 4$, is given by

$$
\begin{aligned}
T_{\mu}^{\mathrm{q}, \sin \mu}= & -\frac{L_{\epsilon}}{16 \pi^{2} a^{n}}\left\{( n - 1 ) \sum _ { i } ( \xi _ { i } - \xi _ { n } ) \left[\frac{d^{2}}{d \tau^{2}} \tilde{\mathcal{M}}_{i i}^{2}-(n-2) a H \frac{d}{d \tau} \tilde{\mathcal{M}}_{i i}^{2}\right.\right. \\
& \left.\left.+\frac{1}{2}\left((n-2)^{2} H^{2}-\frac{n}{n-1} R\right) \tilde{\mathcal{M}}_{i i}^{2}\right]+\sum_{i j} \tilde{\mathcal{M}}_{i j}^{2} \tilde{\mathcal{M}}_{j i}^{2}\right\}
\end{aligned}
$$


We have to add the appropriate counterterms which have the form (see Eqs. (2.6) and (3.46))

$$
\begin{aligned}
T_{\mu}^{\mathrm{q}, \mathrm{ct} \mu}= & \frac{1}{a^{n}}\left\{2(n-1) \sum_{i} \delta \xi_{i}\left[\tilde{\phi}_{i}^{\prime}-\frac{1}{2}(n-2) a H \tilde{\phi}_{i}\right]^{2}\right. \\
& +2(n-1) \sum_{i} \delta \xi_{i} \tilde{\phi}_{i} \tilde{\phi}_{i}^{\prime \prime} \\
& \left.+n\left[\frac{1}{2} \sum_{i} \delta m_{i}^{2} \tilde{\phi}_{i}^{2}+\frac{1}{4} \sum_{i j} \delta \lambda_{i j} a^{4-n} \tilde{\phi}_{i}^{2} \tilde{\phi}_{j}^{2}+\delta \tilde{\Lambda}\right]\right\} \\
& +\delta \tilde{Z} G_{\mu}^{\mu}+\delta \tilde{\alpha}^{(1)} H_{\mu}^{\mu} .
\end{aligned}
$$

We again find, using REDUCE, that the divergences cancel in the sum of $T_{\mu}^{\mathrm{q}, \operatorname{sing} \mu}$ and $T_{\mu}^{\mathrm{q}, \mathrm{ct} \mu}$. There remain finite terms, however, as for $T_{t t}$. These are given by

$$
\begin{aligned}
& T_{\mu}^{\mathrm{q}, \text { add } \mu}=\lim _{n \rightarrow 4}\left(T_{\mu}^{\mathrm{q}, \sin \mu}+T_{\mu}^{\mathrm{q}, \mathrm{ct} \mu}\right)=-\frac{1}{32 \pi^{2} a^{4}} \sum_{i, j} \tilde{\mathcal{M}}_{i j}^{2} \tilde{\mathcal{M}}_{j i}^{2} \\
& -\frac{1}{96 \pi^{2} a^{4}} \sum_{i}\left[\frac{d^{2} \tilde{\mathcal{M}}_{i i}^{2}}{d \tau^{2}}-2 a H \frac{d \tilde{\mathcal{M}}_{i i}^{2}}{d \tau}+2 a^{2} H^{2} \tilde{\mathcal{M}}_{i i}^{2}\right] \\
& -\frac{1}{16 \pi^{2}} \sum_{i}\left(\xi_{i}-\frac{1}{6}\right)\left[12 \frac{H}{a^{3}} \frac{d \tilde{\mathcal{M}}_{i i}^{2}}{d \tau}+\frac{1}{a^{2}}\left(R-18 H^{2}\right) \tilde{\mathcal{M}}_{i i}^{2}-\frac{1}{18} R^{2}-\frac{1}{36}{ }^{(1)} H_{\mu}^{\mu}\right]
\end{aligned}
$$

These agree, for the single-field case, i.e., $N=1$, with Eq. (7.28) of Ref. [36], except for a misprint there: $\left(R+18 H^{2}\right)$ should read $\left(R-18 H^{2}\right)$, as here. The expanded form (7.29) in Ref. [36] is correct. We again refrain from presenting such an expanded form.

The anomalous part is obtained as before

$$
\begin{aligned}
T_{\mu}^{\mathrm{ano} \mu} & =\lim _{n \rightarrow 4} \frac{N}{2880 \pi^{2}} L_{\epsilon}\left({ }^{(2)} H_{\mu}^{\mu}-H_{\mu}^{\mu}\right) \\
& =\frac{N}{2880 \pi^{2}}\left(\frac{R^{\prime \prime}}{a^{2}}+2 H \frac{R^{\prime}}{a}+2 R H^{2}-12 H^{4}\right) .
\end{aligned}
$$

Finally, we have to add the classical part, Eq. (3.39), with $n=4$. So we finally have

$$
T_{\mu}^{\mathrm{ren} \mu}=\left.T_{\mu}^{\mathrm{cl} \mu}\right|_{n=4}+T_{\mu}^{\mathrm{q}, \text { fin } \mu}+T_{\mu}^{\mathrm{q}, \text { add } \mu}+T_{\mu}^{\mathrm{ano} \mu} .
$$




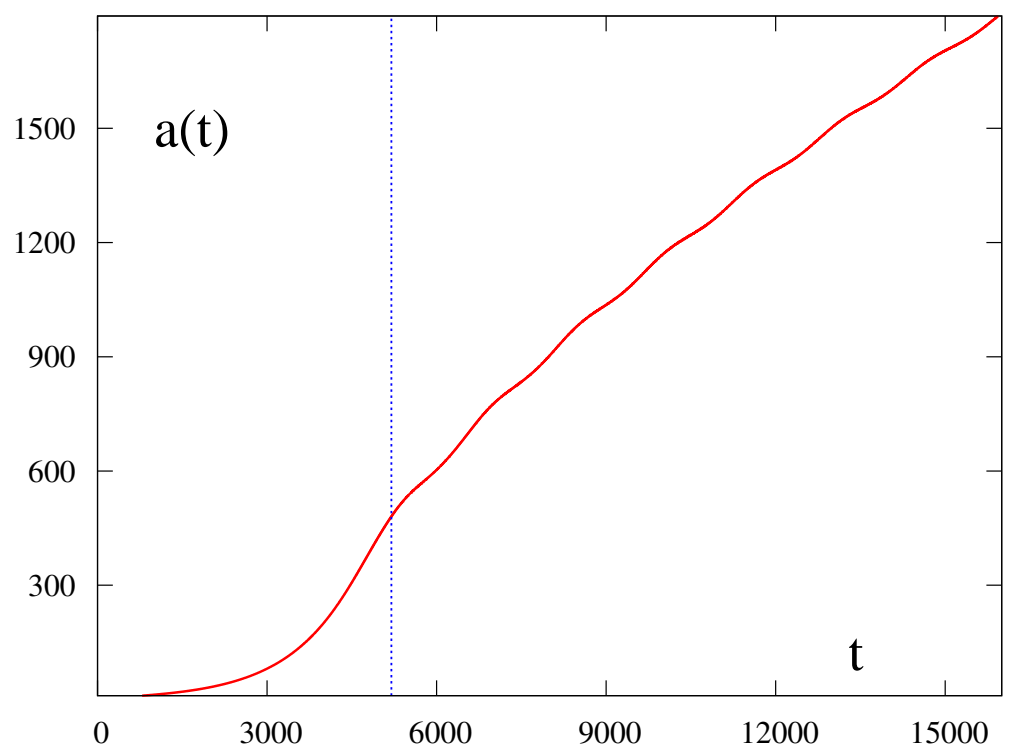

Figure 1: The evolution of the scale parameter $a(t)$ for set I.

\section{Simulations: the hybrid model}

While we have discussed here the renormalisation of a relatively general model of $\mathrm{N}$ coupled fields, the original incentive of our investigation was the application to the hybrid model of inflation. In that case we have a system of only two fields and we can write the Lagrangian as

$$
\begin{aligned}
\mathcal{L}= & \sqrt{-g}\left\{\frac{1}{2} g^{\mu \nu} \partial_{\mu} \phi \partial_{\nu} \phi+\frac{1}{2} g^{\mu \nu} \partial_{\mu} \chi \partial_{\nu} \chi-\frac{1}{2} m^{2} \phi^{2}-\frac{1}{4} \alpha\left(\chi^{2}-v^{2}\right)^{2}\right. \\
& \left.-\frac{1}{2} \lambda \phi^{2} \chi^{2}-\frac{1}{12} R\left(\phi^{2}+\chi^{2}\right)\right\},
\end{aligned}
$$

Here $v$ is the vacuum expectation value of $\chi$. Furthermore, we have to make use of a bare cosmological constant $\tilde{\Lambda}=\alpha v^{4}$ so that the minimum of the classical potential has the value zero. Even when choosing the $\overline{M S}$ prescription for the renormalisation of the masses and couplings we have to make sure that the zero point of the quantum fluctuations at the minimum vanishes, so we have to add a suitable finite counterterm $\delta \tilde{\Lambda}$. Of course this is "fine tuning" and represents the practical aspect of the cosmological constant problem. We have restricted our simulations to the case $\xi_{1}=\xi_{2}=1 / 6$, the conformal couplings. This considerably simplifies the dynamics, and this choice would be natural in a supergravity theory. 


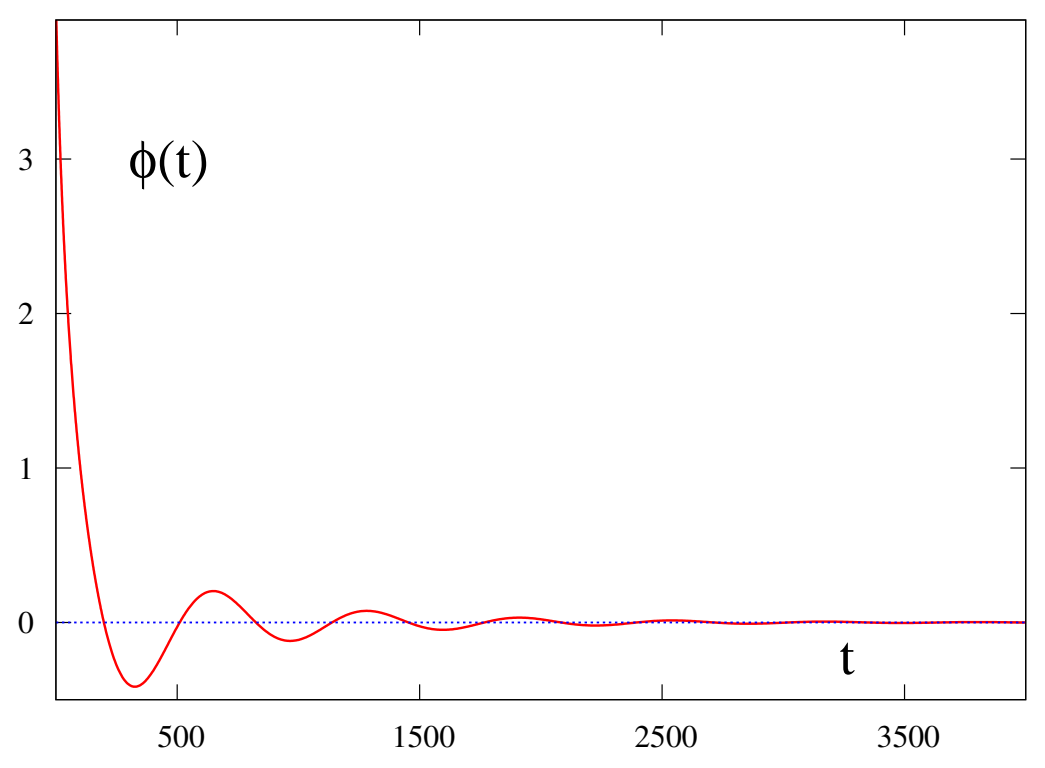

Figure 2: The evolution of the inflation field $\phi(t)$ for set I.

As parameter set $I$ we choose (in Planck units) $v=1, m=0.01, \lambda=10^{-5}$, $\alpha=10^{-5}$. The initial values of the fields are $\phi(0)=4$ and $\chi(0)=0.01$. In Fig. 1 we show the evolution of the scale parameter $a(t)$. We see an initial exponential expansion, followed by a power behaviour $a \simeq t^{0.66}$ as expected for a matter dominated universe. The exponential behaviour is not due to a slow roll of the "inflaton" field $\phi$ which oscillates with a rapidly decreasing amplitude, see Fig. 2, Rather it is the field $\chi$, displayed in Fig. 3 that takes some time before falling from the metastable maximum to the stable minimum. Quantum fluctuations build up after the field $\chi$ starts to fall from $\chi=0$ to $\chi=v=1$. Of course both the classical as well as the quantum energy density decrease with the expansion of the universe. We therefore plot in Fig. 4 the ratio $\rho^{\mathrm{f}} / \rho$ of the fluctuation energy and the total energy densities. The fact that the classical energy density is not converted entirely into fluctuation energy is typical for the one-loop approximation in which the classical fields and the fluctuations remain coherently coupled.

The covariant energy conservation, Eq. (2.17) with $n=4$, is fulfilled with a relative error less than $1 \mathrm{ppm}$.

We also display, in Figs. 5 and 6, the integrands of the fluctuation integrals $\mathcal{F}_{11}$ and $\mathcal{F}_{22}$, including the factor $k^{2} /\left(2 \pi^{2}\right)$. For $\mathcal{F}_{11}$ we display the unsubtracted integrand, the integrand with the zeroth order term removed, and fully subtracted with zeroth and first order in $\mathcal{V}$ removed, see Eq. (4.10). 


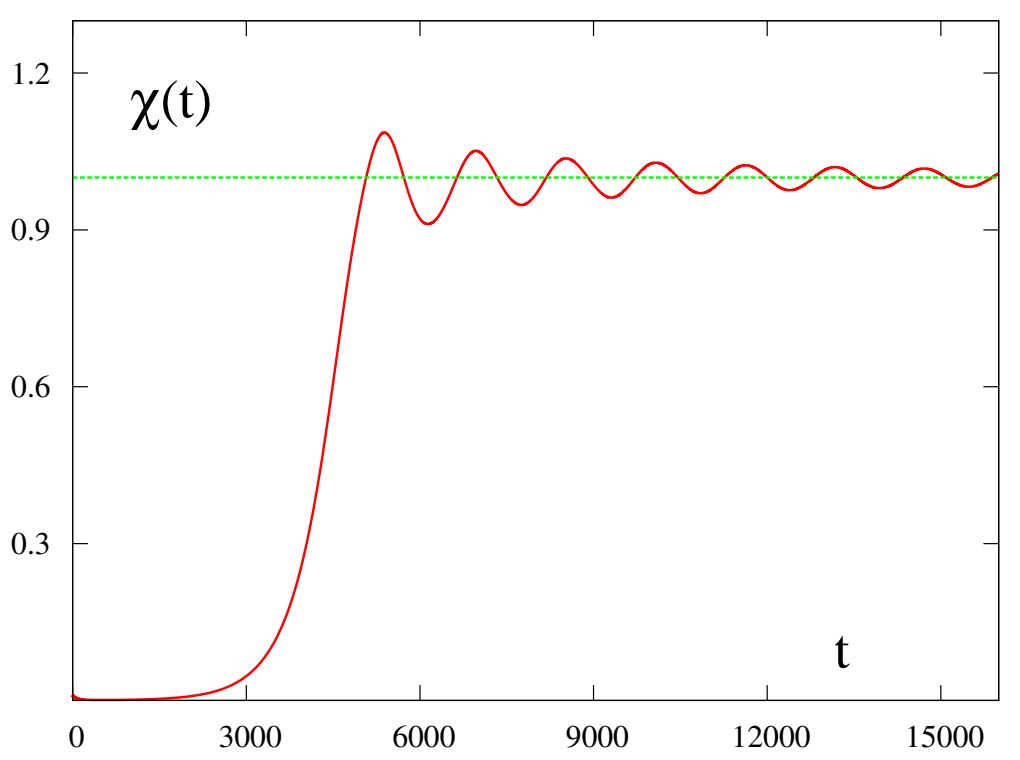

Figure 3: The evolution of the waterfall field $\chi(t)$ for set I.

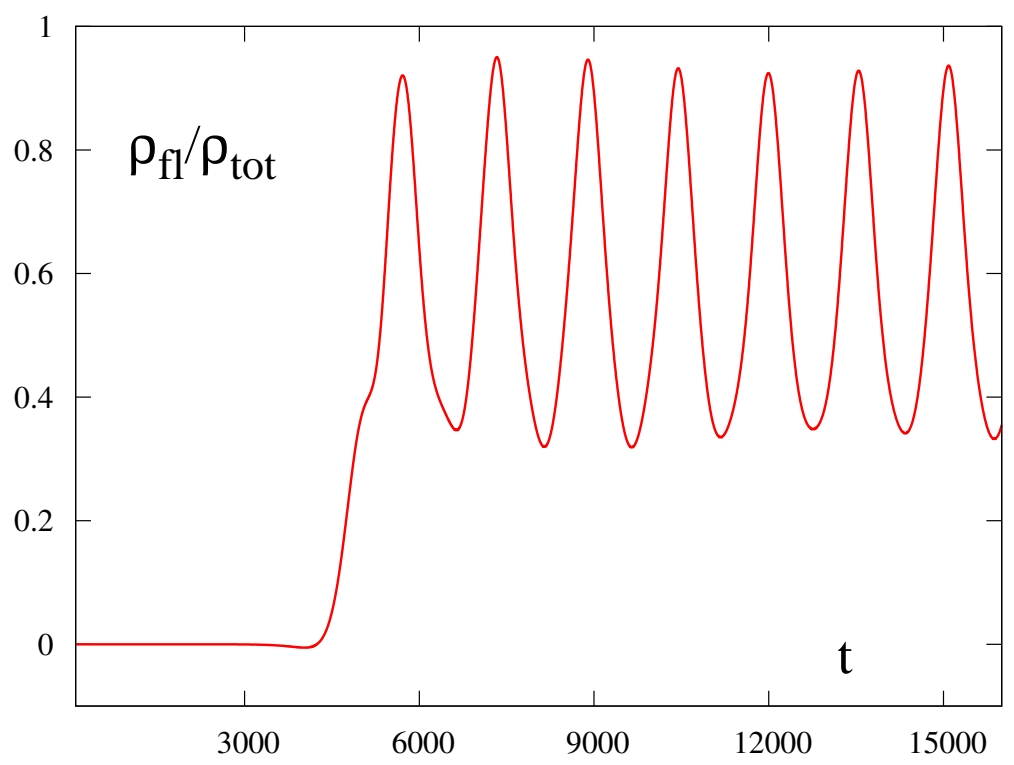

Figure 4: Ratio of fluctuation energy density to total energy density for set I. 

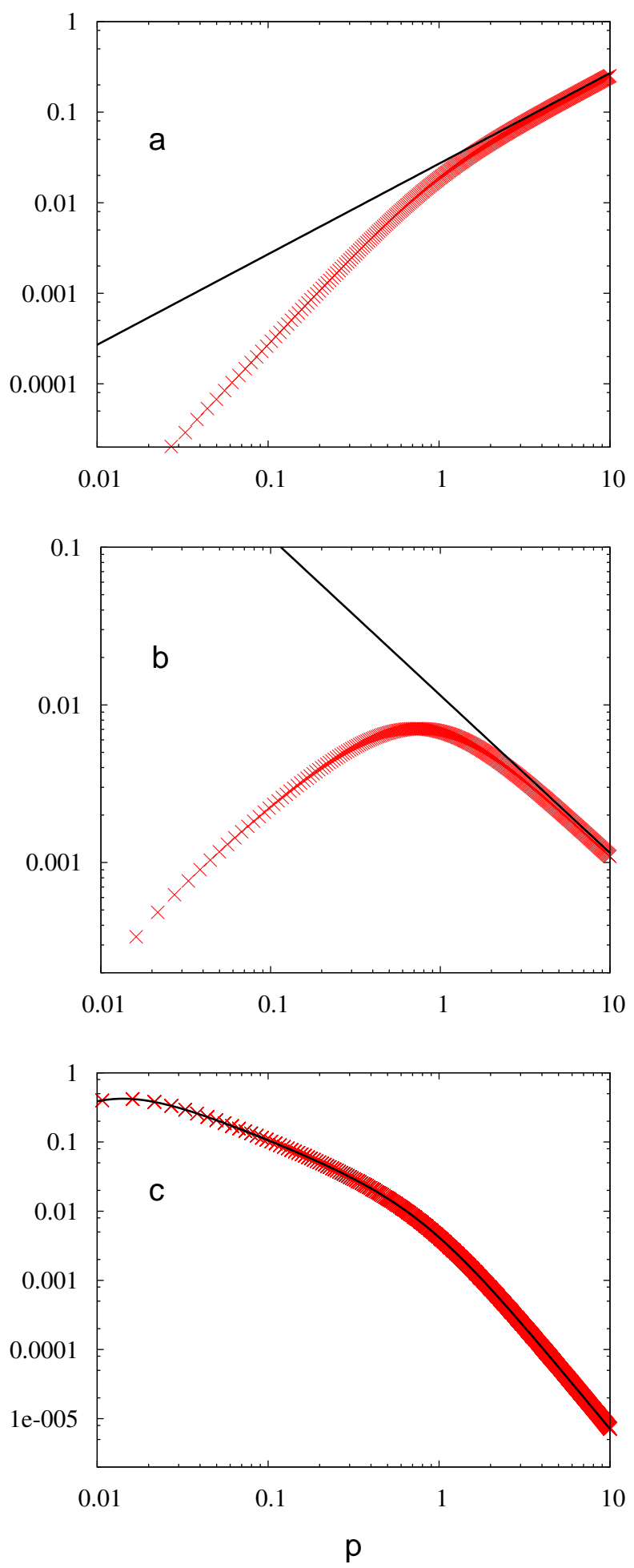

Figure 5: The integrand of the fluctuation integral $\mathcal{F}_{11}$ : (a) without subtraction; (b) after removing the leading term; and (c) with zeroth and first order in $\mathcal{V}$ subtracted, respectively, for set I; crosses: the numerical results; solid lines: in parts a and $\mathrm{b}$ these indicate the asymptotic behaviour $p$ and $p^{-1}$, respectively; in part $\mathrm{c}$ the solid line reproduces the approximation Eq. (G.8). 


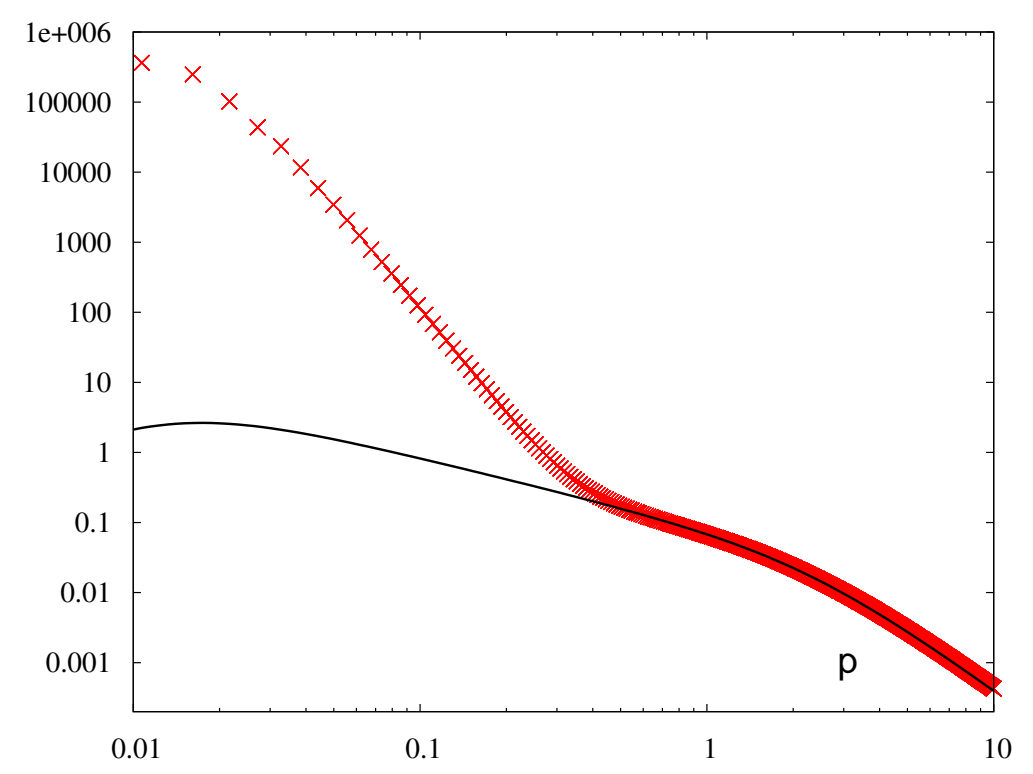

Figure 6: The integrand of the fluctuation integral $\mathcal{F}_{22}$ for set I at $t=6306$; the crosses are the numerical results, while the solid line is the asymptotic approximation Eq. (G.8).

The subtraction is actually performed using the functions $h_{i}^{\alpha}$ introduced in Appendix $\mathrm{C}$ in order to avoid small numerical differences. At large momenta the integrands are seen to behave as $p, p^{-1}$ and $p^{-3}$ respectively. For the fully subtracted integrand we compare the numerical result with the approximate formula (G.8). The agreement is almost perfect: the time at which this integrand is extracted is $t=3154$. There the field $\phi$ has reached its asymptotic value already and corrections due to the field $\chi$ are small. The integrand for $\mathcal{F}_{22}$ is plotted at $t=6306$. There the field $\chi$ has come close to its asymptotic value 1, but is still oscillating around it. Here the approximate formula (G.8) describes well the asymptotic behaviour, but one notices a strong peak at low momenta $p \lesssim .1$. This is in agreement with the fact that a significant part of the classical energy has been converted into fluctuation energy, as already displayed in Fig. 4. Obviously it is the waterfall field whose fluctuations at low momenta have been strongly excited.

\section{Conclusions}

We have presented here a general analysis of the renormalisation of a set of coupled scalar fields in a spatially flat FRW universe with non-minimal 
gravitational coupling and higher curvature gravity terms in the one-loop approximation. By a suitable subtraction procedure we obtain the equations of motion for the classical fields and the energy-momentum tensor in renormalised form. The latter appears of course on the right-hand side of the Friedmann equations. The counterterms are found in the standard form of quantum field theory, they do not depend on numerical cutoffs nor on the initial conditions. The expressions to be evaluated on the computer are finite from the outset, in particular all the subtracted integrals are finite and numerically well-behaved.

The analysis of the divergences used here differs from the procedure used often in the context of quantum field theory in curved space: the adiabatic subtraction, using the eikonal expansion. We have not seen this approach being applied to a coupled channel system; as far as we see this meets problems of principle: while ordinary functions and their derivatives commute, this does not hold for matrix valued functions. Our renormalisation procedure does not suffer from these shortcoming and can be applied to the general case of coupled scalar fields. We expect it to be easily generalised to contain fermionic fields as well and be viable also for supersymmetric models.

The example of coupled scalar fields that has attracted most attention is the hybrid model of inflation. We have now implemented this renormalisation procedure for this specific case and obtained first encouraging results: the energy-momentum conservation is recovered in our scheme to high precision and our asymptotic formulae for the divergences describe the divergent fluctuation integrals with very good accuracy. In the renormalisation procedure we obtain additional finite terms, some of which arise from the $n$ dimensional FRW setting and have no 4D counterpart. These terms are in some cases large and time-dependent, containing $\ln (a(\tau))$, but they cancel corresponding time-dependent terms in the fluctuation integrals and they are therefore crucial for obtaining consistent results, as is discussed in Appendix G.

We have shown for a very simple case that our renormalisation procedure is solid and gives very stable numerical results. We plan to extend our study to the inflationary phase and to different hybrid models in a future publication.

\section{Acknowledgements}

One of us (N.K.) thanks the Humboldt Foundation for financial support, and the Deutsche Elektronensynchrotron DESY, Hamburg, for hospitality. LC acknowledges the support of the DFG under the Collaborative Research Centre 676. 


\section{A Tensors in n-dimensional flat FRW geometry}

The extension of the FRW geometry to $n$ dimensions is done in a specific way: the three-dimensional space of FRW geometry is conformal to threedimensional Minkowski space; it is this three-dimensional space that we extend to $3-\epsilon=n-1$ dimensions in the same way as it is done in the usual dimensional continuation.

For the Christoffel symbols one finds

$$
\Gamma_{i j}^{t}=a \dot{a} \delta_{i j} \quad ; \quad \Gamma_{k t}^{j}=\Gamma_{t k}^{j}=\frac{\dot{a}}{a} \delta_{k}^{j} .
$$

The non-vanishing components of the Riemann tensor are

$$
\begin{aligned}
& R_{i t k}^{t}=\ddot{a} a \delta_{i k} \quad, \quad R_{i j t}^{t}=-\ddot{a} a \delta_{i j}, \\
& R_{t t k}^{l}=\frac{\ddot{a}}{a} \delta_{k}^{l} \quad, \quad R_{t j t}^{l}=-\frac{\ddot{a}}{a} \delta_{j}^{l},
\end{aligned}
$$

for those of the Ricci tensor one finds

$$
R_{t t}=(n-1) \frac{\ddot{a}}{a} \quad, \quad R_{i j}=\left[-\ddot{a} a-(n-2) \dot{a}^{2}\right] \delta_{i j} .
$$

This leads to the Ricci curvature scalar

$$
R=2(n-1) \frac{\ddot{a}}{a}+(n-1)(n-2)\left(\frac{\dot{a}}{a}\right)^{2} .
$$

Expressed in terms of Hubble's constant

$$
H(t)=\frac{\dot{a}(t)}{a(t)}
$$

it takes the form

$$
R=(n-1)\left(2 \dot{H}+n H^{2}\right)
$$

The relevant components of the Einstein tensor become

$$
\begin{aligned}
G_{t t} & =-\frac{(n-1)(n-2)}{2} H^{2}, \\
G_{\mu}^{\mu} & =-\frac{n-2}{2} R .
\end{aligned}
$$


by

The time-time components and the trace of the tensors ${ }^{(n)} H_{\mu \nu}$ are given

$$
\begin{aligned}
{ }^{(1)} H_{t t}= & -6 H \dot{R}+\frac{1}{2} R^{2}-6 H^{2} R \\
& +(n-4)\left(-2 H \dot{R}-(n+1) R H^{2}\right) \\
{ }^{(2)} H_{t t}= & -2 H \dot{R}+\frac{1}{6} R^{2}-2 H^{2} R+(n-4)\left(-\frac{1}{2} H \dot{R}\right. \\
& \left.-\frac{R^{2}}{24(n-1)}-\frac{1}{4}(n+2) H^{2} R+\frac{1}{8}(n-1)(n-2)^{2} H^{4}\right), \\
H_{t t}= & -2 H \dot{R}+\frac{1}{6} R^{2}-2 H^{2} R \\
& +(n-4)\left(-\frac{R^{2}}{6(n-1)}-H^{2} R+\frac{1}{2}(n-1)(n-2) H^{4}\right),
\end{aligned}
$$

${ }^{(1)} H_{\mu}^{\mu}=-6 \ddot{R}-18 H \dot{R}$

$$
+(n-4)\left(-2 \ddot{R}-2(n+2) H \dot{R}-\frac{1}{2} R^{2}\right),
$$

${ }^{(2)} H_{\mu}^{\mu}=-2 \ddot{R}-6 H \dot{R}+(n-4)\left(-\frac{1}{2} \ddot{R}-\frac{1}{2}(n+3) H \dot{R}\right.$

$$
\begin{aligned}
& \left.-\frac{n R^{2}}{8(n-1)}+\frac{1}{4}(n-2)^{2} H^{2} R-\frac{1}{8} n(n-1)(n-2)^{2} H^{4}\right), \\
H_{\mu}^{\mu}= & -2 \ddot{R}-6 H \dot{R}+(n-4)\left(-2 H \dot{R}-\frac{R^{2}}{2(n-1)}\right.
\end{aligned}
$$

$$
\left.+(n-2) H^{2} R-\frac{1}{2} n(n-1)(n-2) H^{4}\right) \text {. }
$$

\section{B Removing the initial singularity}

The initial conditions we have discussed in Sec. 3.2 lead after renormalisation to singularities in the fluctuation integrals when $\tau \rightarrow 0$. They contain terms proportional to $\dot{\mathcal{V}}_{\alpha \beta}(0)$ and $\ddot{\mathcal{V}}_{\alpha \beta}(0)$ which behave as $\tau \ln \left[\left(m_{\alpha 0}+m_{\beta 0}\right) \tau\right]$ and $\tau^{2} \ln \left[\left(m_{\alpha 0}+m_{\beta 0}\right) \tau\right]$ respectively. This does not affect the equations of motion, but the energy-momentum tensor, which contains the first and the second derivatives of the fluctuation integrals, that are infinite as time goes to zero. This means that these initial singularities will appear in the Friedmann equations as well. In Ref. [43] it was shown how to remove such singularities 
by a modification of the initial state. Here we will just briefly repeat these results.

The general concept of the Bogoliubov transformation supposes the replacement of the naive initial state by a transformed vacuum state, annihilated by a superposition of annihilation $a_{\alpha}(\mathbf{k})$ and creation $a_{\alpha}^{+}(-\mathbf{k})$ operators. For this purpose first we define the transformation

$$
\tilde{a}_{\alpha}(\mathbf{k})=\sum_{\beta} \sqrt{\frac{\Omega_{\alpha 0}}{\Omega_{\beta 0}}}\left[C^{\alpha \beta} a_{\beta}(\mathbf{k})-S^{\alpha \beta} a_{\beta}^{+}(-\mathbf{k})\right]
$$

and then a new vacuum state $\mid \tilde{0}>$

$$
\tilde{a}_{\gamma}(\mathbf{k})\left|\tilde{0}>=\sqrt{\frac{\Omega_{\gamma 0}}{\Omega_{\alpha 0}}} C^{\gamma \alpha}\left[a_{\alpha}(\mathbf{k})-\sqrt{\frac{\Omega_{\alpha 0}}{\Omega_{\beta 0}}} \rho_{\alpha \beta} a_{\beta}^{+}(-\mathbf{k})\right]\right| \tilde{0}>=0,
$$

with $\rho=C^{-1} S$ symmetric matrix. By means of the expectation value of $a_{\alpha}(\mathbf{k}) a_{\beta}^{+}\left(\mathbf{k}^{\prime}\right)$ in the new vacua we define a matrix $M_{\alpha \beta}$

$$
<\tilde{0}\left|a_{\alpha}(\mathbf{k}) a_{\beta}^{+}\left(\mathbf{k}^{\prime}\right)\right| \tilde{0}>=(2 \pi)^{2} \delta^{3}\left(\mathbf{k}-\mathbf{k}^{\prime}\right) 2 \sqrt{\Omega_{\alpha 0} \Omega_{\beta 0}} M_{\alpha \beta}(k),
$$

which is related to the matrix $\rho$ as follows

$$
M-\rho M^{T} \rho^{+}=I .
$$

Following the schema presented in Appendix A of [43] one sees that the cancellation of the dangerous terms we have mentioned above requires

$$
\begin{aligned}
\operatorname{Im} \rho^{\alpha \beta} & =\frac{1}{2 \Omega_{\alpha 0} \Omega_{\beta 0}} \frac{1}{\left(\Omega_{\alpha 0}+\Omega_{\beta 0}\right)^{2}} \mathcal{V}_{\alpha \beta}^{\prime}(0), \\
\operatorname{Re} \rho^{\alpha \beta} & =\frac{1}{2 \Omega_{\alpha 0} \Omega_{\beta 0}} \frac{1}{\left(\Omega_{\alpha 0}+\Omega_{\beta 0}\right)^{3}} \mathcal{V}_{\alpha \beta}^{\prime \prime}(0) .
\end{aligned}
$$

So after $\rho$ is known we have to determine the fluctuation integrals and the energy-momentum tensor in the Bogoliubov-transformed vacuum. But for the numerical implementation it is more convenient to redefine the mode functions

$$
\tilde{f}_{i}^{\alpha}(\tau ; \boldsymbol{k})=\sum_{\beta} \sqrt{\frac{\Omega_{\alpha 0}}{\Omega_{\beta 0}}}\left[f_{i}^{\beta}(\tau ; \boldsymbol{k}) N_{\beta \alpha}+f_{i}^{\beta *}(\tau ; \boldsymbol{k}) \rho^{\beta \gamma *} N_{\gamma \alpha}\right],
$$

where the matrix $N$ is defined as $N \times N=M$. Here $\tilde{f}_{i}^{\alpha}$ are solutions of the same mode equations as $f_{i}^{\alpha}$ are. For the fluctuation integrals and the energy-momentum tensor the expressions presented in Sec. 3.3 remain valid, with the mode functions $f_{i}^{\alpha}$ replaced by the Bogoliubov transformed ones $\tilde{f}_{i}^{\alpha}$. 


\section{Perturbative Expansion}

The perturbative expansion of the mode functions for the case of coupled system in curved space-time follows a scheme introduced in Ref. [37]. We split the mode functions $f_{i}^{\alpha}(\tau ; k)$ into the free part and higher order terms represented by the reduced mode functions $h_{i}^{\alpha}(\tau ; k)$ with the ansatz

$$
f_{i}^{\alpha}(\tau ; k)=e^{-i \Omega_{\alpha 0} \tau}\left[f_{i 0}^{\alpha}+h_{i}^{\alpha}(\tau ; k)\right] .
$$

The mode equations (3.25) are equivalent to the following integral equation

$$
f_{i}^{\alpha}(\tau ; k)=e^{-i \Omega_{\alpha 0} \tau} f_{i 0}^{\alpha}+\int_{0}^{\tau} d \tau^{\prime} \Delta_{i j}\left(\tau-\tau^{\prime} ; k\right) \mathcal{V}_{j k}\left(\tau^{\prime}\right) f_{k}^{\alpha}\left(\tau^{\prime} ; k\right)
$$

with the potential $\mathcal{V}_{i j}$ introduced in Eq. (3.44) and with the retarded kernel of the free equation

$$
\Delta_{i j}\left(\tau-\tau^{\prime} ; k\right)=\sum_{\beta} \frac{i}{2 \Omega_{\beta 0}} \Theta\left(\tau-\tau^{\prime}\right) f_{i 0}^{\beta} f_{j 0}^{\beta}\left[e^{i \Omega_{\beta 0}\left(\tau-\tau^{\prime}\right)}-e^{-i \Omega_{\beta 0}\left(\tau-\tau^{\prime}\right)}\right] .
$$

Then the reduced mode functions satisfy the integral equation

$$
\begin{aligned}
h_{i}^{\alpha}(\tau ; k)= & \int_{0}^{\tau} d \tau^{\prime} \sum_{\beta} \frac{i}{2 \Omega_{\beta 0}} f_{i 0}^{\beta} f_{j 0}^{\beta}\left[e^{i\left(\Omega_{\alpha 0}+\Omega_{\beta 0}\right)\left(\tau-\tau^{\prime}\right)}-e^{i\left(\Omega_{\alpha 0}-\Omega_{\beta 0}\right)\left(\tau-\tau^{\prime}\right)}\right] \\
& \times \mathcal{V}_{j k}\left(\tau^{\prime}\right)\left[f_{k 0}^{\alpha}+h_{k}^{\alpha}\left(\tau^{\prime} ; k\right)\right]
\end{aligned}
$$

and the corresponding differential equations become

$$
h_{i}^{\alpha \prime \prime}-2 i \Omega_{\alpha 0} h_{i}^{\alpha \prime}(\tau ; k)=-\sum_{j}\left[\mathcal{V}_{i j} f_{j 0}^{\alpha}+\left(\tilde{\mathcal{M}}_{i j}^{2}-m_{\alpha 0}^{2} \delta_{i j}\right) h_{j}^{\alpha}(\tau ; k)\right] \text {. }
$$

The initial conditions (3.33) and (3.34) imply $h_{i}^{\alpha}(0 ; k)=h_{i}^{\alpha \prime}(0 ; k)=0$.

Now we expand the reduced mode functions $h_{i}^{\alpha}$ with respect to orders in potential $\mathcal{V}_{i j}$

$$
h_{i}^{\alpha}(\tau)=h_{i}^{\alpha(1)}(\tau)+h_{i}^{\alpha(2)}(\tau)+h_{i}^{\alpha(3)}(\tau)+\ldots
$$

where $h_{i}^{\alpha(n)}$ denotes the $n$th order in $\mathcal{V}_{i j}$. For our calculations we will need only the first and second terms of this expansion, therefore we introduce notation $h_{i}^{\alpha \overline{(n)}}(\tau)$ for the sum over all orders in $\mathcal{V}_{i j}$ starting with the $n$th order, i.e. $h_{i}^{\alpha \overline{(1)}}=h_{i}^{\alpha(1)}+h_{i}^{\alpha \overline{(2)}}$. Using the iteration one can obtain reduced 
mode functions of any order. In first order we have

$$
\begin{aligned}
h_{i}^{\alpha(1)}(\tau ; k)= & \sum_{\beta} \frac{i}{2 \Omega_{\beta 0}} f_{i 0}^{\beta} f_{j 0}^{\beta} f_{k 0}^{\alpha}\left[\int_{0}^{\tau} d \tau^{\prime} \mathcal{V}_{j k}\left(\tau^{\prime}\right) e^{i\left(\Omega_{\alpha 0}+\Omega_{\beta 0}\right)\left(\tau-\tau^{\prime}\right)}\right. \\
& \left.-\int_{0}^{\tau} d \tau^{\prime} \mathcal{V}_{j k}\left(\tau^{\prime}\right) e^{i\left(\Omega_{\alpha 0}-\Omega_{\beta 0}\right)\left(\tau-\tau^{\prime}\right)}\right]
\end{aligned}
$$

The large-momentum behaviour of the Fourier-type integrals is analysed in Appendix D, To leading order in $1 / k$ we find

$$
\operatorname{Re} h_{i}^{\alpha(1)}(\tau ; k) \simeq \sum_{\beta} \frac{f_{i 0}^{\beta} \tilde{\mathcal{V}}_{\beta \alpha}(\tau)}{2 \Omega_{\beta 0}\left(\Omega_{\alpha 0}+\Omega_{\beta_{0}}\right)}
$$

and

$$
\operatorname{Im} h_{i}^{\alpha(1)}(\tau ; k) \simeq-\sum_{\beta} \frac{1}{2 \Omega_{\beta 0}} \int_{0}^{\tau} d \tau^{\prime} f_{i 0}^{\beta} \tilde{\mathcal{V}}_{\beta \alpha}\left(\tau^{\prime}\right),
$$

where we have introduced the notation

$$
f_{j 0}^{\beta} f_{k 0}^{\alpha} \mathcal{V}_{i j}=\tilde{\mathcal{V}}_{\beta \alpha}
$$

$\tilde{\mathcal{V}}_{\alpha \beta}$ is symmetric in the indices, as is $\mathcal{V}_{j k}$.

For the fluctuation integrals (3.36) we need to compute

$$
\begin{aligned}
\operatorname{Re}\left(f_{i}^{\alpha} f_{j}^{\alpha *}\right)= & f_{i 0}^{\alpha} f_{j 0}^{\alpha}+f_{i 0}^{\alpha} \operatorname{Re} h_{j}^{\alpha}+f_{j 0}^{\alpha} \operatorname{Re} h_{i}^{\alpha}+\operatorname{Re}\left(h_{i}^{\alpha} h_{j}^{\alpha *}\right) \\
= & f_{i 0}^{\alpha} f_{j 0}^{\alpha}+f_{i 0}^{\alpha} \operatorname{Re} h_{j}^{\alpha(1)}+f_{j 0}^{\alpha} \operatorname{Re} h_{i}^{\alpha(1)}+f_{i 0}^{\alpha} \operatorname{Re} h_{j}^{\alpha(\overline{2})} \\
& +f_{j 0}^{\alpha} \operatorname{Re} h_{i}^{\alpha(\overline{2})}+\operatorname{Re}\left(h_{i}^{\alpha(\overline{1})} h_{j}^{\alpha(\overline{1}) *}\right) .
\end{aligned}
$$

For large momenta the first term is constant, of course. The second and third terms behave as $1 / k^{2}$, see Eq. (C.8). The term $\operatorname{Re}\left(h_{i}^{\alpha(\overline{1})} h_{j}^{\alpha(\overline{1}) *}\right)$ obviously contains $\operatorname{Im} h_{i}^{\alpha(1)} \operatorname{Im} h_{j}^{\alpha(1)}$ which likewise behaves as $1 / k^{2}$, see Eq. (C.91). It would imply a nonlocal divergence. This contribution can be shown to cancel against a similar term in $f_{i 0}^{\alpha} \operatorname{Re} h_{j}^{\alpha(2)}+f_{j 0}^{\alpha} \operatorname{Re} h_{i}^{\alpha(2)}$, however. For the single field case this has been discussed in Ref. [37] (see there below Eq. (56)).

The second and third term appear in the integrand of fluctuation integral as:

$$
\begin{aligned}
& \sum_{\alpha} \frac{1}{2 \Omega_{\alpha 0}}\left[f_{i 0}^{\alpha} \operatorname{Re} h_{j}^{\alpha(1)}+f_{j 0}^{\alpha} \operatorname{Re} h_{i}^{\alpha(1)}\right] \\
& \simeq-\sum_{\alpha, \beta} f_{i 0}^{\alpha} f_{j 0}^{\beta} \frac{\tilde{\mathcal{V}}_{\beta \alpha}}{2 \Omega_{\alpha 0} \Omega_{\beta 0}\left(\Omega_{\alpha 0}+\Omega_{\beta 0}\right)},
\end{aligned}
$$


where we have used Eq. (C.8).

We have already used the Wronski relation at $\tau=0$ when defining the initial conditions in Sec. 3.2. Now we will consider the Wronskian in general, which will be useful for simplifying the mode integrals occurring in the energy-momentum tensor

$$
W=f_{i}^{\alpha *} f_{i}^{\beta \prime}-f_{i}^{\alpha * \prime} f_{i}^{\beta}=-2 i \Omega_{\alpha 0} \delta_{\alpha \beta} .
$$

From this we obtain the relation

$$
i\left(\Omega_{\alpha 0}+\Omega_{\beta 0}\right)\left(f_{i 0}^{\alpha} h_{i}^{\beta}+f_{i 0}^{\beta} h_{i}^{\alpha *}+h_{i}^{\alpha *} h_{i}^{\beta}\right)=f_{i 0}^{\alpha} h_{i}^{\beta \prime}-f_{i 0}^{\beta} h_{i}^{\alpha * \prime}+h_{i}^{\alpha *} h_{i}^{\beta \prime}-h_{i}^{\alpha * \prime} h_{i}^{\beta} .
$$

For our purposes we will need only the $\alpha=\beta$ part of this relation, i.e.:

$$
\Omega_{\alpha 0}\left(2 f_{i}^{\alpha} \operatorname{Re} h_{i}^{\alpha}+\left|h_{i}^{\alpha}\right|^{2}\right)=f_{i 0}^{\alpha} \operatorname{Im} h_{i}^{\alpha \prime}+\operatorname{Im} h_{i}^{\alpha *} h_{i}^{\alpha \prime} .
$$

This relation can be used to simplify the kinetic term in the quantum part of the $t t$ component of the energy-momentum tensor. Using the expansion in terms of reduced mode functions and Eq. C.15) one finds

$$
\sum_{\alpha} \frac{f_{i}^{\alpha^{\prime}} f_{i}^{\alpha * \prime}}{2 \Omega_{\alpha 0}}=\sum_{\alpha} \frac{1}{2 \Omega_{\alpha 0}}\left[\Omega_{\alpha 0}^{2}\left(f_{i 0}^{\alpha 2}-2 f_{i 0}^{\alpha} \operatorname{Re} h_{i}^{\alpha}-\left|h_{i}^{\alpha}\right|^{2}\right)+\left|h_{i}^{\alpha \prime}\right|^{2}\right] .
$$

This can be combined with

$$
\sum_{\alpha} \frac{f_{i}^{\alpha} f_{i}^{\alpha *}}{2 \Omega_{\alpha 0}}=\sum_{\alpha} \frac{1}{2 \Omega_{\alpha 0}}\left[f_{i 0}^{\alpha 2}+2 f_{i 0}^{\alpha} \operatorname{Re} h_{i}^{\alpha}+\left|h_{i}^{\alpha}\right|^{2}\right]
$$

to obtain

$$
\sum_{\alpha} \frac{1}{2 \Omega_{\alpha 0}}\left[f_{i}^{\alpha \prime} f_{i}^{\alpha * \prime}+\Omega_{\alpha 0}^{2} f_{i}^{\alpha} f_{i}^{\alpha *}\right]=\sum_{\alpha} \frac{1}{2 \Omega_{\alpha 0}}\left[2 \Omega_{\alpha 0}^{2} f_{i 0}^{\alpha 2}+\left|h_{i}^{\alpha \prime}\right|^{2}\right] .
$$

When integrated over momentum the first term in the bracket, including the prefactor $1 / 2 \Omega_{\alpha 0}$ leads to a quartic divergence. The second term is dominated at large momenta by the square of the imaginary part of $h_{i}^{\alpha(1)^{\prime}}$ (see Eq. (C.9)):

$$
\frac{1}{2 \Omega_{\alpha 0}}\left|h_{i}^{\alpha \prime}\right|^{2} \simeq \frac{1}{2 \Omega_{\alpha 0}} \operatorname{Im}\left(h_{i}^{\alpha \prime}\right)^{2} \simeq \sum_{\beta} \frac{1}{8 \Omega_{\alpha 0} \Omega_{\beta 0}^{2}} \tilde{\mathcal{V}}_{\beta \alpha} \tilde{\mathcal{V}}_{\alpha \beta}
$$

up to terms of order $k^{-4}$. This implies a logarithmic divergence. Therefore the subleading terms are not important, and we may replace this asymptotic estimate by

$$
\frac{1}{2 \Omega_{\alpha 0}}\left|h_{i}^{\alpha \prime}\right|^{2} \simeq \sum_{\beta} \frac{1}{4 \Omega_{\alpha 0} \Omega_{\beta 0}\left(\Omega_{\alpha 0}+\Omega_{\beta 0}\right)} \tilde{\mathcal{V}}_{\beta \alpha} \tilde{\mathcal{V}}_{\alpha \beta} .
$$

This has the advantage that it can be integrated in closed form and is of the same form as other logarithmically divergent contribution, see Eq. (C.12). 


\section{Large-momentum behaviour of a Fourier transform}

When analysing the reduced mode function $h_{i}^{\alpha(1)}$, Eq. (C.7), at large momentum we encounter the expression

$$
i \int_{0}^{\tau} d \tau^{\prime} \mathcal{V}_{j k}\left(\tau^{\prime}\right)\left[e^{i\left(\Omega_{\alpha 0}+\Omega_{\beta 0}\right)\left(\tau-\tau^{\prime}\right)}-e^{i\left(\Omega_{\alpha 0}-\Omega_{\beta 0}\right)\left(\tau-\tau^{\prime}\right)}\right]
$$

For $\alpha=\beta$ this integral has been analysed for large $k$ in Ref. [37]. For $\alpha \neq \beta$ the analysis is somewhat more involved. The first exponential $\exp \left[i\left(\Omega_{\alpha 0}+\Omega_{\beta 0}\right)\left(\tau-\tau^{\prime}\right)\right]$ will oscillate strongly when $k \rightarrow \infty$. So this part of the integral will be dominated by the region around $\tau=\tau^{\prime}$, i.e., the upper end of the integration interval. The large-momentum behaviour can be analysed by repeated integrations by parts, as for the case $\alpha=\beta$, see below. The second exponential $\exp \left[i\left(\Omega_{\alpha 0}-\Omega_{\beta 0}\right)\left(\tau-\tau^{\prime}\right)\right]$ will approach unity as $k \rightarrow \infty$. Indeed

$$
\Omega_{\alpha 0}-\Omega_{\beta 0}=\frac{m_{\alpha 0}^{2}-m_{\beta 0}^{2}}{\Omega_{\alpha 0}+\Omega_{\beta 0}} \simeq \frac{m_{\alpha 0}^{2}-m_{\beta 0}^{2}}{k}
$$

as $k \rightarrow \infty$, so the exponent goes to zero in this limit.

Considering the two parts separately we find for the first one, via integrations by parts,

$$
\begin{aligned}
& i \int_{0}^{\tau} d \tau^{\prime} \mathcal{V}_{j k}\left(\tau^{\prime}\right) e^{i\left(\Omega_{\alpha 0}+\Omega_{\beta 0}\right)\left(\tau-\tau^{\prime}\right)} \\
& =-\frac{1}{\Omega_{\alpha 0}+\Omega_{\beta 0}} \mathcal{V}_{j k}(\tau) \\
& +\frac{i}{\left(\Omega_{\alpha 0}+\Omega_{\beta 0}\right)^{2}}\left[\mathcal{V}_{j k}^{\prime}(\tau)-\mathcal{V}_{j k}^{\prime}(0) e^{i\left(\Omega_{\alpha 0}+\Omega_{\beta 0}\right) \tau}\right]+\ldots
\end{aligned}
$$

and for the second one, by expanding the exponential,

$$
\begin{aligned}
& -i \int_{0}^{\tau} d \tau^{\prime} \mathcal{V}_{j k}\left(\tau^{\prime}\right) e^{i\left(\Omega_{\alpha 0}-\Omega_{\beta 0}\right)\left(\tau-\tau^{\prime}\right)} \\
& =-i \int_{0}^{\tau} d \tau^{\prime} \mathcal{V}_{j k}\left(\tau^{\prime}\right)-\frac{m_{\alpha 0}^{2}-m_{\beta 0}^{2}}{\Omega_{\alpha 0}+\Omega_{\beta 0}} \int_{0}^{\tau} d \tau^{\prime} \mathcal{V}_{i j}\left(\tau^{\prime}\right)\left(\tau-\tau^{\prime}\right) \\
& +i \frac{\left(m_{\alpha 0}^{2}-m_{\beta 0}^{2}\right)^{2}}{2\left(\Omega_{\alpha 0}+\Omega_{\beta 0}\right)^{2}} \int_{0}^{\tau} d \tau^{\prime} \mathcal{V}_{i j}\left(\tau^{\prime}\right)\left(\tau-\tau^{\prime}\right)^{2}+\ldots
\end{aligned}
$$

When contracted with $f_{j 0}^{\beta} f_{k 0}^{\alpha}$ the second term on the right hand side does not contribute as $\tilde{\mathcal{V}}_{\alpha \beta}=f_{j 0}^{\beta} f_{k 0}^{\alpha} \mathcal{V}_{j k}$ is symmetric in $\alpha$ and $\beta$. 


\section{E Dimensionally regulated integrals}

For the dimensional regularisation we need some identities $(D=4-\epsilon)$ :

$$
\begin{aligned}
& \int \frac{d^{D-1} k}{(2 \pi)^{D-1}} \frac{1}{2 \Omega_{\alpha 0} \Omega_{\beta 0}\left(\Omega_{\alpha 0}+\Omega_{\beta 0}\right)} \\
& =\frac{1}{16 \pi^{2}}\left[L_{\epsilon}-\ln \frac{m_{\alpha 0}^{2}}{\mu^{2}}+1+\frac{m_{\beta 0}^{2}}{m_{\alpha 0}^{2}-m_{\beta 0}^{2}} \ln \frac{m_{\beta 0}^{2}}{m_{\alpha 0}^{2}}\right] \\
& \int \frac{d^{D-1} k}{(2 \pi)^{D-1}} \frac{1}{2 \Omega_{\alpha 0}}=-\frac{m_{\alpha 0}^{2}}{16 \pi^{2}}\left[L_{\epsilon}-\ln \frac{m_{\alpha 0}^{2}}{\mu^{2}}+1\right] \\
& \int \frac{d^{D-1} k}{(2 \pi)^{D-1}} \Omega_{\alpha 0}=-\frac{m_{\alpha 0}^{4}}{32 \pi^{2}}\left[L_{\epsilon}-\ln \frac{m_{\alpha 0}^{2}}{\mu^{2}}+\frac{3}{2}\right]
\end{aligned}
$$

where $L_{\epsilon}=\frac{2}{\epsilon}-\gamma+\ln 4 \pi$.

In the limit $m_{\beta 0}^{2} \rightarrow m_{\alpha 0}^{2}$ the finite terms in the first of these equations reduce to

$$
\lim _{m_{\beta 0}^{2} \rightarrow m_{\alpha 0}^{2}}\left[-\ln \frac{m_{\alpha 0}^{2}}{\mu^{2}}+1+\frac{m_{\beta 0}^{2}}{m_{\alpha 0}^{2}-m_{\beta 0}^{2}} \ln \frac{m_{\beta 0}^{2}}{m_{\alpha 0}^{2}}\right]=-\ln \frac{m_{\alpha 0}^{2}}{\mu^{2}} .
$$

\section{F Counterterms}

Using the explicit form of $\tilde{\mathcal{M}}_{i j}^{2}$ the counterterm Lagrangian Eq. (4.5) can be decomposed into the usual mass, coupling constant and other counterterms as

$$
\begin{aligned}
\mathcal{L}^{\mathrm{ct}}=-\frac{1}{2}\left(\delta m_{i}^{2}+\right. & \left.\delta \xi_{i} R\right) a^{2} \tilde{\phi}_{i}^{2}-\frac{a^{4-n}}{4} \delta \lambda_{i j} \tilde{\phi}_{i}^{2} \tilde{\phi}_{j}^{2} \\
& +a^{n}\left(\frac{1}{2} \delta \tilde{Z} R+\frac{1}{2} \delta \tilde{\alpha} R^{2}-\delta \tilde{\Lambda}\right) .
\end{aligned}
$$

Note that the first terms do not have a prefactor $a^{n}$, as it has been incorporated into the rescaled fields $\tilde{\phi}_{i}$. 
When we compare this to the expansion of the right hand side of Eq. (4.5) we find

$$
\begin{aligned}
\delta m_{i}^{2} & =\frac{1}{16 \pi^{2}} L_{\epsilon}\left[3 \lambda_{i i} m_{i}^{2}+\sum_{j \neq i} \lambda_{i j} m_{j}^{2}\right] \\
\delta \xi_{i} & =\frac{1}{16 \pi^{2}} L_{\epsilon}\left[3 \lambda_{i i}\left(\xi_{i}-1 / 6\right)+\sum_{j \neq i} \lambda_{i j}\left(\xi_{j}-1 / 6\right)\right] \\
\delta \lambda_{i i} & =\frac{1}{16 \pi^{2}} L_{\epsilon}\left[9 \lambda_{i i}^{2}+\sum_{j \neq i} \lambda_{i j}^{2}\right] \\
\delta \lambda_{i j} & =\frac{1}{16 \pi^{2}} L_{\epsilon} \lambda_{i j}\left[3 \lambda_{i i}+3 \lambda_{j j}+4 \lambda_{i j}\right] \\
\delta \tilde{Z} & =-\frac{1}{16 \pi^{2}} L_{\epsilon} \sum_{i}\left(\xi_{i}-1 / 6\right) m_{i}^{2} \\
\delta \tilde{\alpha} & =-\frac{1}{32 \pi^{2}} L_{\epsilon} \sum_{i}\left(\xi_{i}-1 / 6\right)^{2} \\
\delta \tilde{\Lambda} & =\frac{1}{64 \pi^{2}} L_{\epsilon} \sum_{i} m_{i}^{4} .
\end{aligned}
$$

These are in agreement with the counterterms found for the case of a single field [36]. We recall that the last three renormalisation constants are related to renormalisation of the left-hand side of Einstein's equations, which have been redefined as renormalisation of the energy-momentum tensor, see Eqs. (2.5) and (2.6).

The counterterms do not by themselves define the renormalisation. Choosing them as given above defines the $\overline{M S}$ scheme. They can be modified by finite terms in order that the renormalised theory satisfies certain requirements. E.g., if we want our universe to become matter or radiation dominated at late times we will have to ensure that the effective cosmological constant remains zero. We will also want Newton's constant to retain its observed value. The details of such finite renormalisation depend on the specific model and usually involve tedious calculations. We do not address this issue here.

\section{G An instructive example}

The fluctuation integrals obviously play a central rôle in the one-loop approximation to non-equilibrium dynamics. In particular it plays a rôle for fixing 
the parameters in the equilibrium theory, one would not like the renormalisation conditions to be dependent on the expansion of the universe. As we have mentioned already the $\ln a(\tau)$ - term in the finite parts $\mathcal{F}_{i j}^{\mathrm{ft}}$ is new here and suggests a logarithmic increase. It can become very large if the universe expands by many $e$-foldings. So it is interesting to analyse the behaviour of the subtracted integral $\mathcal{F}_{i j}^{\text {sub }}$ as well. We will do this for just one field, and setting for simplicity $\xi=1 / 6$. Furthermore we will assume that the universe has ended up, at least approximately, in its ground state, i.e. that the classical field $\phi$ has reached its equilibrium value, which we assume to be 0 and that the fluctuations have almost died out, in a sense we will specify shortly.

The mode equation for $f(k, \tau)$ is given by

$$
f^{\prime \prime}+\left(k^{2}+\tilde{\mathcal{M}}^{2}(\tau)\right) f=0 .
$$

If the classical field has reached its minimum $\mathcal{M}^{2}$ is still time dependent

$$
\tilde{\mathcal{M}}^{2}(\tau) \simeq m^{2} a^{2}
$$

so the modes are still under the influence of a time-depending "potential". At least for large $k$ we can approximately solve the mode equation using the semiclassical approximation

$$
f(k, \tau) \simeq \mathrm{C}(\mathrm{k})\left(k^{2}+\mathcal{M}^{2}(\tau)\right)^{-1 / 4} \exp ( \pm i S),
$$

with

$$
S=\int_{\tau_{0}}^{\tau} d \tau^{\prime} \sqrt{k^{2}+\tilde{\mathcal{M}}^{2}\left(\tau^{\prime}\right)}
$$

So we have

$$
|f(k)|^{2} \simeq \frac{C^{2}(k)}{\sqrt{k^{2}+\tilde{\mathcal{M}}^{2}(\tau)}} .
$$

We will fix the constant $C(k)$ by the assumption that the system is, at least as far as the high frequency modes are concerned, already in its adiabatic vacuum at $\tau_{0}$. Then for sufficiently large $k$

$$
C^{2}(k) \simeq \sqrt{k^{2}+\tilde{\mathcal{M}}^{2}\left(\tau_{0}\right)} .
$$

Then with $\tilde{\mathcal{M}}^{2}=m^{2} a^{2}$ and $\mathcal{M}^{2}\left(\tau_{0}\right)=m_{0}$ we have

$$
\mathcal{V}(\tau)=m^{2} a^{2}(\tau)-m 0^{2}
$$

and the integrand of subtracted fluctuation integral becomes

$$
\frac{k^{2}}{4 \pi^{2}}\left[\frac{1}{\sqrt{k^{2}+m^{2} a^{2}}}-\frac{1}{\sqrt{k^{2}+m_{0}^{2}}}+\frac{m^{2} a^{2}-m_{0}^{2}}{2 \sqrt{k^{2}+m_{0}^{2}}}\right] .
$$


Integrating from $k=0$ to infinity we obtain

$$
\mathcal{F}^{\text {sub }} \simeq \frac{1}{16 \pi^{2}}\left(m^{2} a^{2} \ln \frac{a^{2} m^{2}}{m_{0}^{2}}-m^{2} a^{2}+m_{0}^{2}\right),
$$

with corrections due to the low-energy modes. The finite term $\mathcal{F}^{\mathrm{ft}}$ here is given by

$$
\mathcal{F}^{\mathrm{ft}}=\frac{1}{16 \pi^{2}}\left[m^{2} a^{2} \ln \frac{m_{0}^{2}}{\mu^{2} a^{2}}-m_{0}^{2}\right],
$$

where we have used the single-field version of Eq. (4.11) For the finite value $\mathcal{F}^{\text {fin }}$ we therefore get

$$
\mathcal{F}^{\mathrm{fin}}=\mathcal{F}^{\mathrm{ft}}+\mathcal{F}^{\mathrm{sub}} \simeq \frac{m^{2} a^{2}}{16 \pi^{2}}\left(\ln \frac{m^{2}}{\mu^{2}}-1\right) .
$$

The logarithmic dependence on $a$ has disappeared. The factor $a^{2}$ is natural. In the equation of motion the fluctuation integral adds to $\tilde{\phi}^{2}=a^{2} \phi^{2}$.

As an application of interest we may consider the broken symmetry case. We have a Higgs field $\chi$, with a classical vacuum expectation value $v$. If its self-coupling is denoted by $\alpha$ and if we use the $\overline{M S}$ scheme the one-loop corrected equilibrium position is determined by

$$
<\tilde{\chi}^{2}>=v^{2} a^{2}+\alpha \mathcal{F}^{\mathrm{fin}} .
$$

With Eq. (G.11) we see that the expectation value of $\chi$ itself becomes independent of $a$, as it should. Were it not for the $\ln a$ term in $\mathcal{F}^{\mathrm{ft}}$, the vacuum expectation value would shift continuously with the expansion of the universe.

Of course there are corrections, but this is to be expected. In particular, if the fluctuations have thermalised, the vacuum expectation value of the Higgs field becomes a function of temperature. So our estimate would be strictly valid only after the temperature has reached 0 .

We would like to add that the heuristic estimates for the behaviour of the quantum fluctuations which we have used here are corroborated by numerical simulations. Actually they may be used for estimating the high momentum contribution to the subtracted fluctuation integral.

\section{References}

[1] A. Ringwald, Z. Phys. C34, 481 (1987).

[2] D. Boyanovsky, H. J. de Vega and R. Holman, Phys. Rev. D49, 2769 (1994), [hep-ph/9310319]. 
[3] D. Boyanovsky, D. Cormier, H. J. de Vega, R. Holman and S. P. Kumar, Phys. Rev. D57, 2166 (1998), [hep-ph/9709232].

[4] D. Boyanovsky et al., Phys. Rev. D56, 1939 (1997), [hep-ph/9703327].

[5] S. A. Ramsey and B. L. Hu, Phys. Rev. D56, 678 (1997), [hep$\mathrm{ph} / 9706207]$.

[6] J. Baacke and C. Patzold, Phys. Rev. D62, 084008 (2000), [hep$\mathrm{ph} / 9912505]$.

[7] A. D. Linde, Phys. Lett. B249, 18 (1990).

[8] A. D. Linde, Phys. Lett. B259, 38 (1991).

[9] A. D. Linde, Phys. Rev. D49, 748 (1994), [astro-ph/9307002].

[10] J. Garcia-Bellido and A. D. Linde, Phys. Rev. D57, 6075 (1998), [hep$\mathrm{ph} / 9711360]$.

[11] D. H. Lyth and A. Riotto, Phys. Rept. 314, 1 (1999), [hep-ph/9807278].

[12] J. Garcia-Bellido, D. Y. Grigoriev, A. Kusenko and M. E. Shaposhnikov, Phys. Rev. D60, 123504 (1999), [hep-ph/9902449].

[13] M. Bastero-Gil, S. F. King and J. Sanderson, Phys. Rev. D60, 103517 (1999), [hep-ph/9904315].

[14] L. M. Krauss and M. Trodden, Phys. Rev. Lett. 83, 1502 (1999), [hep$\mathrm{ph} / 9902420]$.

[15] G. N. Felder et al., Phys. Rev. Lett. 87, 011601 (2001), [hep$\mathrm{ph} / 0012142]$.

[16] R. Micha and M. G. Schmidt, Eur. Phys. J. C14, 547 (2000), [hep$\mathrm{ph} / 9908228]$.

[17] G. N. Felder, L. Kofman and A. D. Linde, Phys. Rev. D64, 123517 (2001), [hep-th/0106179].

[18] E. J. Copeland, D. Lyth, A. Rajantie and M. Trodden, Phys. Rev. D64, 043506 (2001), [hep-ph/0103231].

[19] W. Buchmuller, L. Covi and D. Delepine, Phys. Lett. B491, 183 (2000), [hep-ph/0006168]. 
[20] H. P. Nilles, M. Peloso and L. Sorbo, JHEP 04, 004 (2001), [hepth/0103202].

[21] T. Asaka, W. Buchmuller and L. Covi, Phys. Lett. B510, 271 (2001), [hep-ph/0104037].

[22] D. Cormier, K. Heitmann and A. Mazumdar, Phys. Rev. D65, 083521 (2002), [hep-ph/0105236].

[23] J. Garcia-Bellido, M. Garcia Perez and A. Gonzalez-Arroyo, Phys. Rev. D67, 103501 (2003), [hep-ph/0208228].

[24] S. Borsanyi, A. Patkos and D. Sexty, Phys. Rev. D66, 025014 (2002), [hep-ph/0203133].

[25] S. Borsanyi, A. Patkos and D. Sexty, Phys. Rev. D68, 063512 (2003), [hep-ph/0303147].

[26] R. Allahverdi, R. Brandenberger, F.-Y. Cyr-Racine and A. Mazumdar, 1001.2600.

[27] J. Baacke and A. Heinen, Phys. Rev. D69, 083523 (2004), [hep$\mathrm{ph} / 0311282]$.

[28] F. L. Bezrukov and M. Shaposhnikov, Phys. Lett. B659, 703 (2008), [0710.3755].

[29] N. Kaloper, L. Sorbo and J. Yokoyama, Phys. Rev. D78, 043527 (2008), [0803.3809].

[30] A. De Simone, M. P. Hertzberg and F. Wilczek, Phys. Lett. B678, 1 (2009), [0812.4946].

[31] F. L. Bezrukov, A. Magnin and M. Shaposhnikov, Phys. Lett. B675, 88 (2009), [0812.4950].

[32] J. Garcia-Bellido, D. G. Figueroa and J. Rubio, Phys. Rev. D79, 063531 (2009), [0812.4624].

[33] M. Bordag, J. Lindig and V. M. Mostepaneko, Class. Quant. Grav. 15, 581 (1998).

[34] J. Lindig, Phys. Rev. D59, 064011 (1999), [hep-th/9808133].

[35] J. Baacke, K. Heitmann and C. Patzold, Phys. Rev. D56, 6556 (1997), [hep-ph/9706274]. 
[36] J. Baacke and C. Patzold, Phys. Rev. D61, 024016 (1999), [hepph/9906417].

[37] J. Baacke, K. Heitmann and C. Patzold, Phys. Rev. D55, 2320 (1997), [hep-th/9608006].

[38] N. Birrell and P. Davies, Quantum fields in curved space (Cambridge University Press, Cambridge, U.K., 1982).

[39] E. Calzetta and B. L. Hu, Phys. Rev. D35, 495 (1987).

[40] J. S. Schwinger, J. Math. Phys. 2, 407 (1961).

[41] L. V. Keldysh, Zh. Eksp. Teor. Fiz. 47, 1515 (1964).

[42] J. Baacke, K. Heitmann and C. Patzold, Phys. Rev. D57, 6398 (1998), [hep-th/9711144].

[43] J. Baacke and N. Kevlishvili, Phys. Rev. D81, 023509 (2010), [0910.1128].

[44] A. Hearn, REDUCE User's Manual Version 3.8 (Anthony.C. Hearn, Santa Monica, CA, USA, 2004). 